\title{
A Cluster Expansion Approach to a One-Dimensional Boltzmann Equation: A Validity Result
}

\author{
S. Caprino ${ }^{1}$, M. Pulvirenti ${ }^{2}$ \\ 1 Dipartimento di Matematica Università di L'Aquila, Italy \\ 2 Dipartimento di Matematica Università di Roma, La Sapienza, Italy
}

Received: 13 September 1993/in revised form: 31 January 1994

\begin{abstract}
We consider a stochastic particle system on the line and prove that, when the number of particles diverges and the probability of a collision is suitably rescaled, the system is well described by a one-dimensional Boltzmann equation. The result holds globally in time, without any smallness assumption.
\end{abstract}

\section{Contents}

1. Introduction . . . . . . . . . . . . . . . . . 603

2. The Model . . . . . . . . . . . . . . . . . . . . . . . . . . . . . . 605

3. Considerations on the Model and Preliminary Estimates . . . . . . . . . . . . . 607

4. The Main Result and the Strategy of the Proof . . . . . . . . . . . . . . . . . 614

5. Proof of Proposition 4.1 c. . . . . . . . . . . . . . . . . . . . . . . . . . . . . . . . . . . . . . . 616

6. From Small to Large $\lambda$. . . . . . . . . . . . . . . . . . . . . . . . . . . . . 624

\section{Introduction}

It is well known how hard the problem is of proving rigorously the validity of the Boltzmann equation for a rarefield gas from the Newton equations of a system of $N$ interacting particles. Very few results are available up to now (see [1] and [2] in the References). The only successful way to approach this problem is to introduce the BBGKY hierarchy for the $j$-particle distribution functions and try to show that they converge, in the Boltzmann-Grad limit, to a factorizing solution of the Boltzmann hierarchy (that is a $j$-fold tensor product of the solution of the Boltzmann equation), provided that the initial state of the BBGKY hierarchy converges to a factorizing state. This program can be achieved completely once one has suitable $L_{\infty}$ estimates on the $j$-particle distribution functions for the $N$-particle system. However this is a very difficult task in general. In [1] and [2] such estimates have been obtained for a system of hard balls in special situations: either for a short time or for a moderate perturbation of the vacuum. For more general situations, the problem of proving $L_{\infty}$ estimates for 
the $j$-particle distribution functions is much harder than that of proving $L_{\infty}$ bounds for the solutions of the Boltzmann equation itself. This is not yet known (maybe even false) although recent progress on the existence problem for the Boltzmann equation have been achieved by compactness methods [3].

In this paper we prove the validity of a one-dimensional kinetic Boltzmann equation starting from a $N$-particle system. The existence theory for such a kinetic equation is well understood (see e.g. [4] and references quoted therein) and, in particular, $L_{\infty}$ bounds for the solutions are available. The underlying one-dimensional model we are considering is stochastic: two particles on the line collide with probability $\varepsilon$ and go ahead with probability $1-\varepsilon$. In this way the number of collisions per unit time performed by a tagged particle should be finite in the limit $N \rightarrow \infty$ (if $\varepsilon \sim N^{-1}$ ). Notice that the stochasticity of the model involves only the collision mechanism and this is strictly pointwise. In other words the stochasticity we allow is the minimal one we need to give sense to a one dimensional problem. On the other hand the model we consider is the simplest one with these features. A model of this type has been considered, at the level of a kinetic picture, by Ianiro and Lebowitz for some stationary problem [5].

We now anticipate the leading idea in the validity proof. The $j$-particle distribution functions satisfy a hierarchy of equations (with the same structure as the BBGKY hierarchy for Hamiltonian systems) from which it is extremely hard to obtain $L_{\infty}$ estimates globally in time. However for any (tagged) group of $j$-particles we can prove, for the combination $\lambda=\varepsilon N$ small, the following remarkable fact: with a large probability, each particle interacts with a finite cluster of other particles and such clusters are disjoint. As a consequence the $j$-particle distribution function is expressed as a $j$-fold tensor product of $L_{\infty}$ positive functions plus a correction, which is small in $L_{1}$. This fact, combined with a trace control, allows us to prove the convergence of the one-particle distribution function to the solution of our onedimensional Boltzmann equation and also the propagation of chaos. The tool we use to outline the above picture is a cluster expansion technique similar to those used in Equilibrium Statistical Mechanics. The analysis is perturbative (with respect to the free motion) and therefore the cluster expansion is converging for small values of $\lambda$, independently of the size of the initial datum. Moreover, we can control the local particle density. This implies, thanks to the finite speed of propagation, that any particle is influenced, in a small interval of time, by a small fraction of the total number of molecules. Hence the analysis for small $\lambda$ can be applied locally in space and time to give the desired result by an inductive procedure.

Beyond the purpose of the present paper, the cluster dynamics we propose is interesting in itself and clarify the mechanism of propagation of chaos which is expected to hold in a rarefield gas. It is conceivable that the cluster dynamics is the real physical mechanism responsible for the factorization of the $j$-particle distribution functions in the limit $N \rightarrow \infty$, also for Hamiltonian systems, although this crucial features would be very difficult to prove.

We also mention a recent result in which the validity of the two-dimensional Broadwell model was established, assuming the existence of a smooth solution and starting from a stochastic particle system on a lattice [6]. The techniques of that paper do not apply to the present context because they were based on a lattice approximation and on the presence of a noise on the free stream. Our model is less stochastic, as we said, but is one-dimensional and we take advantage from this fact. Actually the approach presented here is not directly applicable to solve the problem posed in [6].

The paper is organized as follows. In Sect. 2 we present the model, develop some preliminary heuristic considerations and pose the problem. In Sect. 3 we discuss some 
preliminary steps concerning our particle model. In Sect. 4 we present and discuss the convergence result for small $\lambda$. In Sect. 5 we face the crucial step of the paper. Here we develop our cluster expansion and obtain estimates for the $j$-particle distributions already used in Sect. 4. In Sect. 6 we show how to extend the previous results to $\lambda$ large.

We finally remark that our approach is based on probability estimates, namely on the fact that the $j$-particle distribution functions are expectations with respect to a probability measure and that large and small probability events can be estimated differently. Actually it seems very difficult to obtain the same result by a purely PDE approach.

\section{The Model}

Let us consider a system of $N$ particles on the line. Denote by $Z_{N}=\left(X_{N}, V_{N}\right)=$ $\left\{x_{1} v_{1}, \ldots, x_{N} v_{N}\right\}$ a state of the system, where $x_{\imath}$ and $v_{\imath}$ are the position and the velocity of the $i$-th particle and $X_{N} \in \mathbf{R}^{N}$ and $V_{N} \in V^{N}$ denote the set of all positions and velocities. $V=\{ \pm 1, \pm 2\}$ is the velocity space of a single particle. A collision between two particles is defined through the following law: if $v_{1}$ and $v_{2}$ are the ingoing velocities and $v_{1}^{\prime}$ and $v_{2}^{\prime}$ are the outgoing velocities, then two cases are possible

$$
\begin{aligned}
& \text { either }\left(v_{1}, v_{2}\right)=(2,-1) \text { and }\left(v_{1}^{\prime}, v_{2}^{\prime}\right)=(1,-2) \\
& \text { or }\left(v_{1}, v_{2}\right)=(1,-2) \text { and }\left(v_{1}^{\prime}, v_{2}^{\prime}\right)=(2,-1) .
\end{aligned}
$$

If $v_{1}=-v_{2}$ then the particles go ahead freely.

Notice that the above collision rule is equivalent to a specular reflection of each colliding particle. However we prefer a labelling for which the direction of the velocity does not change in the collision because in such a way any pair of particles can interact at most only once. This fact will play an important role later on. Moreover collisions of particles travelling in the same direction are considered as transparent.

The dynamics of the system is stochastic, in the sense that each pair of particles collides (independently) with probability $\varepsilon$ and go ahead with probability $1-\varepsilon$. Such a stochastic process can be represented in the sample space:

$$
\Omega=\{0,1\}^{\frac{N(N-1)}{2}} .
$$

Hence $\omega \in \Omega, \omega=\omega(i, j)$ is a function defined on the set of all pairs of particles, taking values 0 and 1 . The time evolution of the system is described by means of a one-parameter group of transformations

$$
T_{\omega}^{t}:(\mathbf{R} \times V)^{N} \rightarrow(\mathbf{R} \times V)^{N} \quad t \in \mathbf{R}
$$

defined as follows. $T_{\omega}^{t} Z_{N}$ is the free motion up to the first time in which two particles (say $i$ and $j$ ) arrive at the same position, then if $\left|v_{i}-v_{j}\right|=3$ and $\omega(i, j)=1$ (that is we are in a precollisional situation) the two particles collide according to the law given in (2.1), otherwise they keep their free motion. We repeat the procedure up to the next collision time and so on. Notice that the modulus of the relative velocity of any pair of particles $\left|v_{i}-v_{j}\right|$ is an invariant for such dynamics:

$$
\left|v_{i}-v_{j}\right|=\left|v_{\imath}^{\prime}-v_{\jmath}^{\prime}\right|=3 \quad \forall i, j \in N
$$

$T_{\omega}^{t}$ is, in principle, well defined since, by (2.1), two particles having interacted once cannot interact anymore. Moreover $T_{\omega}^{t}$ is almost everywhere (a.e.) defined with respect 
to the Lebesgue measure on $(\mathbf{R} \times V)^{N}$. Indeed it is not defined on the sets of all $Z_{N}$ 's which deliver triple collisions and on the set of configurations for which, for some $i, j \in N$, it is $x_{i}=x_{j},\left|v_{\imath}-v_{j}\right|=3$ and $\omega(i, j)=1$, being, in this last case, not possible to distinguish between pre- and post-collisional configurations: nevertheless these two sets have measure zero.

Defining the probability of a single event $\omega \in \Omega$ by

$$
p(\omega)=\prod_{i, j} \varepsilon^{\omega(i, j)}(1-\varepsilon)^{1-\omega(i, j)},
$$

where $\prod_{i, j}$ denotes the product on all pairs, $\{\Omega, p\}$ is a probability space and $T_{\omega}^{t}$ a stochastic process on it.

Suppose now that the $N$-particle system is described at time zero by a symmetric probability density on $(\mathbf{R} \times V)^{N}$ denoted by $\mu_{0}^{N}$. We define the time evolved density $\mu^{N}(\cdot, t)$ by:

$$
\mu^{N}\left(Z_{N}, t\right)=\sum_{\omega} p(\omega) \mu_{\omega}^{N}\left(Z_{N}, t\right):=\mathbb{E}\left[\mu_{\omega}^{N}\left(Z_{N}, t\right)\right]
$$

where

$$
\mu_{\omega}^{N}\left(Z_{N}, t\right)=\mu_{0}^{N}\left(T_{\omega}^{-t} Z_{N}\right) .
$$

We now want to establish an evolution equation for $\mu_{\omega}^{N}$ and $\mu^{N}$ and to this end we will proceed by heuristic arguments, which will be made rigorous in the next section.

We have, formally, for any sample $\omega \in \Omega$ :

$$
\begin{gathered}
\left(\partial_{t}+\sum_{i=1}^{N} v_{i} \partial_{i}\right) \mu_{\omega}^{N}\left(Z_{N}, t\right)=\frac{3}{2} \sum_{i \neq j} \omega(i, j) \chi(i, j) \\
\delta\left(x_{i}-x_{j}\right)\left\{\mu_{\omega}^{N}\left(Z_{N}^{\prime}(i, j), t\right)-\mu_{\omega}^{N}\left(Z_{N}, t\right)\right\},
\end{gathered}
$$

where $3=\left|v_{i}-v_{j}\right|$ is the relative velocity, $\partial_{\imath}$ is the derivative with respect to $x_{i}$, $\delta(\cdot)$ is the $\delta$-function centered in zero, $\chi(i, j)=1$ if $\left|v_{\imath}-v_{j}\right|=3$ and 0 otherwise and

$$
Z_{N}^{\prime}(i, j)=\left(x_{1} v_{1}, \ldots x_{i} v_{\imath}^{\prime}, \ldots x_{j} v_{\jmath}^{\prime}, \ldots x_{N} v_{N}\right)
$$

Taking now the expectation with respect to $p(\omega)$ in (2.6), we obtain

$$
\begin{aligned}
& \left(\partial_{t}+\sum_{i=1}^{N} v_{i} \partial_{i}\right) \mu^{N}\left(Z_{n}, t\right) \\
& \quad=\frac{3}{2} \varepsilon \sum_{i \neq j} \delta\left(x_{\imath}-x_{j}\right) \chi(i, j)\left\{\mu^{N}\left(Z_{N}^{\prime}(i, j), t\right)-\mu^{N}\left(Z_{N}, t\right)\right\} .
\end{aligned}
$$

This equation is expected to hold because the event $\omega(i, j)$ appearing in (2.6) is independent of the random variable in the curl brackets.

Let us now derive, always formally, the kinetic equation associated to the above process. We start by the usual $j$-particle distribution densities, defined as:

$$
f_{j}^{N}\left(Z_{j}, t\right)=\int \mathrm{d} Z_{N-j} \mu^{N}\left(Z_{N}, t\right)
$$

where $\mathrm{d} Z_{N-j}=\sum_{v_{\jmath+1} \ldots v_{N}} \mathrm{~d} x_{j+1} \ldots \mathrm{d} x_{N}$. 
After a rather straightforward algebra, we obtain from (2.7):

$$
\left(\partial_{t}+\sum_{i=1}^{\jmath} v_{i} \partial_{i}\right) f_{j}^{N}=\varepsilon G_{j} f_{j}^{N}+\varepsilon(N-j) C_{j, j+1} f_{j+1}^{N}
$$

where

$$
G_{\jmath} f_{j}^{N}\left(Z_{j}, t\right)=\frac{3}{2} \sum_{\substack{i \neq k \\ i, k=1 \ldots j}} \delta\left(x_{i}-x_{k}\right) \chi(i, k)\left\{f_{j}^{N}\left(Z_{j}^{\prime}(i, k), t\right)-f_{j}^{N}\left(Z_{j}, t\right)\right\}
$$

and

$$
\begin{aligned}
C_{j, \jmath+1} f_{\jmath+1}^{N}\left(Z_{j}, t\right)= & 3 \sum_{i=1}^{j} \sum_{\substack{v_{j+1} \in V \\
v_{i}-v_{j+1} \mid=3}}\left\{f_{j+1}^{N}\left(x_{1} v_{1}, \ldots x_{i} v_{\imath}^{\prime}, \ldots x_{i} v_{j+1}^{\prime}, t\right)\right. \\
& \left.-f_{j+1}^{N}\left(x_{1} v_{1}, \ldots x_{i} v_{i}, \ldots x_{i} v_{j+1} t\right)\right\} .
\end{aligned}
$$

We are interested in performing the Boltzmann-Grad limit on Eq. (2.11), that is letting $N \rightarrow \infty$ and $\varepsilon \rightarrow 0$ while $N \varepsilon \rightarrow \lambda, \lambda$ a fixed number. Assuming that such limit of the $f_{j}^{N}$ does exist and denoting it by $f_{\jmath}$, these functions are expected to satisfy the following infinite hierarchy of equations:

$$
\left(\partial_{t}+\sum_{i=1}^{j} v_{\imath} \partial_{\jmath}\right) f_{\jmath}=\lambda C_{j, j+1} f_{\jmath+1}
$$

Moreover, if the $f_{j}$ are factorizing functions, i.e.:

$$
f_{j}\left(x_{1} v_{1}, \ldots, x_{j} v_{j}, t\right)=\prod_{k=1}^{\jmath} f\left(x_{k} v_{k}, t\right),
$$

then $f$ solves, as it is easily seen, the Boltzmann equation associated to our model, which is

$$
\left(\partial_{t}+v \partial_{x}\right) f(x, v)=3 \lambda\left\{f\left(x, v^{\prime}\right) f\left(x, v_{1}^{\prime}\right)-f(x, v) f\left(x, v_{1}\right)\right\}
$$

where $v_{1}$ is the only precollisional velocity associated to $v$, that is $\left|v_{1}-v\right|=3$.

The aim of this paper is indeed to prove that all the above arguments can be made rigorous, so that the Boltzmann-Grad limit holds in this context.

\section{Considerations on the Model and Preliminary Estimates}

In this section we shall make rigorous some of the considerations on the process $T_{\omega}^{t}$ developed at a heuristic level so far. Moreover, we shall give two basic estimates which will be employed in the proof of the main result.

We start by introducing the "collision manifold" $\mathscr{F}^{\omega}$ defined, for any $\omega$, as:

$$
\begin{gathered}
\mathscr{F}^{\omega}=\bigcup_{i, \jmath: \omega(i, j)=1} \mathscr{F}_{i \jmath}^{\omega}, \\
\mathscr{F}_{\imath \jmath}^{\omega}=\left\{Z_{N}: x_{i}=x_{j},\left|v_{i}-v_{j}\right|=3, \omega(i, j)=1\right\} .
\end{gathered}
$$


On $\mathscr{F}^{\omega}$ we define the measure $d Y$ whose restriction on $\mathscr{F}_{i j}^{\omega}$ is given by:

$$
d Y=\sum_{v_{1} \ldots v_{N}} \chi(i, j) d x_{1} \ldots d x_{i-1} d x_{i+1} \ldots d x_{j} \ldots d x_{N}
$$

Such codimension-one manifold $\mathscr{F}^{\omega}$ is indeed of great importance as far as we are concerned with the dynamics of our system, so that for a correct definition of the equation describing the evolution in time of $\mu_{\omega}^{N}$, we have to extend the definition of $T_{\omega}^{t}$ on $\mathscr{F}^{\omega}$. For this reason we have to specify whether the velocities of the colliding pairs in the configurations $Z_{N}$ belonging to $\mathscr{F}^{\omega}$ are to be interpreted as incoming or outcoming velocities.

Denoting the free flow semigroup by

$$
S^{t} Z_{N}=\left(x_{1}+v_{1} t, v_{1} \ldots x_{N}+v_{N} t, v_{N}\right),
$$

we define, for any $Y \in \mathscr{F}^{\omega}$ :

$$
T_{\omega}^{t} Y=S^{t} \pi(Y) \quad \text { for } \quad t \in(0, \alpha(Y))
$$

where

$$
\alpha(Y)=\inf \left\{\tau>0: S^{\tau} \pi(Y) \in \mathscr{F}^{\omega}\right\}
$$

and

$$
\pi(Y)=Y^{\prime}(i, j) \quad \text { if } \quad Y \in \mathscr{F}_{i j}^{\omega} .
$$

Definition (3.5) implies that we consider the elements of $\mathscr{F}^{\omega}$ as having precollisional velocities. The set $\bigcup_{0<t<\alpha(Y)} S^{t} \pi(Y)$ is of positive measure so that we can conclude that $T_{\omega}^{t}$ is defined for $d Y$-almost all $Y \in \mathscr{F}^{\omega}$. Furthermore, by (3.5) it follows that

$$
T_{\omega}^{-t} Y=S^{-t} Y \text { for } t \text { small. }
$$

Now we are ready to derive an evolution equation for $\mu_{\omega}^{N}\left(Z_{N}, t\right)$. Notice that, by the previous arguments, we can extend $\mu_{\omega}^{N}(\cdot, t)$ on the collision manifold according to Definition (2.5).

Given $Z_{N}$, let $t_{1}$ be the smallest collision time in the interval $[0, t]$. Then by (3.5)-(3.8):

$$
\begin{aligned}
\mu_{\omega}^{N}\left(Z_{N}, t\right)= & \mu_{0}^{N}\left(T_{\omega}^{-t} Z_{N}\right)=\mu_{0}^{N}\left(T_{\omega}^{-\left(t-t_{1}\right)} T_{\omega}^{-t_{1}} Z_{N}\right)=\mu_{0}^{N}\left(T_{\omega}^{-\left(t-t_{1}\right)} \pi\left(S^{-t_{1}} Z_{N}\right)\right) \\
= & \left\{\mu_{\omega}^{N}\left(\pi\left(S^{-t_{1}} Z_{N}\right), t-t_{1}\right)-\mu_{\omega}^{N}\left(S^{-t_{1}} Z_{N}, t-t_{1}\right)\right\} \\
& +\mu_{\omega}^{N}\left(S^{-t_{1}} Z_{N}, t-t_{1}\right) .
\end{aligned}
$$

If $t_{1} \ldots t_{k}$ are the solutions of the equations $x_{i}-v_{i} t=x_{j}-v_{\jmath} t$ with $\left|v_{i}-v_{\jmath}\right|=3$, $\omega(i, j)=1, i, j=1 \ldots N$ in the interval $(0, t)$, iterating the above procedure we obtain

$$
\begin{aligned}
\mu_{\omega}^{N}\left(Z_{N}, t\right)= & \mu_{0}^{N}\left(S^{-t} Z_{N}\right)+\sum_{r=1}^{k}\left\{\mu_{\omega}^{N}\left(\pi\left(S^{-t_{r}} Z_{N}\right), t-t_{r}\right)-\mu_{\omega}^{N}\left(S^{-t_{r}} Z_{N}, t-t_{r}\right)\right\} \\
= & \mu_{0}^{N}\left(S^{-t} Z_{N}\right)+\frac{3}{2} \sum_{i \neq j} \omega(i, j) \chi(i, j) \int_{0}^{t} \mathrm{~d} \tau\left\{\delta\left(x_{i}-v_{i} \tau-x_{j}+v_{\jmath} \tau\right)\right. \\
& \times\left[\mu_{\omega}^{N}\left(\pi\left(S^{-\tau} Z_{N}, t-\tau\right)-\mu_{\omega}^{N}\left(S^{-\tau} Z_{N}, t-\tau\right)\right]\right\}
\end{aligned}
$$


Integrating now (3.9) against a smooth test function with compact support $\Phi$, we have:

$$
\begin{aligned}
& \int \mathrm{d} Z_{N} \Phi\left(Z_{N}\right) \mu_{\omega}^{N}\left(Z_{N}, t\right)=\int \mathrm{d} Z_{N} \Phi\left(S^{t} Z_{N}\right) \mu_{0}^{N}\left(Z_{N}\right) \\
& +3 \int_{0}^{t} \mathrm{~d} \tau \int_{\mathscr{F} \omega} \mathrm{d} Y \Phi\left(S^{t-\tau} Y\right)\left\{\mu_{\omega}^{N}(\pi(Y), \tau)-\mu_{\omega}^{N}(Y, \tau)\right\} .
\end{aligned}
$$

From (3.10) we deduce that the r.h.s. is an a.e. differentiable function w.r.t. the time and

$$
\begin{aligned}
\frac{d}{d t} \int \mathrm{d} Z_{N} \Phi\left(Z_{N}\right) \mu_{\omega}^{N}\left(Z_{N}, t\right)=\int \mathrm{d} Z_{N} \sum_{i=1}^{N} v_{\imath} \partial_{i} \Phi\left(Z_{N}\right) \mu_{\omega}^{N}\left(Z_{N}, t\right) \\
\quad+3 \int_{\mathscr{F}^{\omega}} \mathrm{d} Y \Phi(Y)\left\{\mu_{\omega}^{N}(\pi(Y), t)-\mu_{\omega}^{N}(Y, t)\right\} \\
=\int \mathrm{d} Z_{N} \sum_{\imath=1}^{N} v_{i} \partial_{i} \Phi\left(Z_{N}\right) \mu_{\omega}^{N}\left(Z_{N}, t\right) \\
\quad+\frac{3}{2} \sum_{i \neq j} \omega(i, j) \int_{\mathscr{F}_{i j}} \mathrm{~d} Y \Phi(Y)\left\{\mu_{\omega}^{N}\left(Y^{\prime}(i, j), t\right)-\mu_{\omega}^{N}(Y, t)\right\}
\end{aligned}
$$

where

$$
\mathscr{F}_{\imath \jmath}=\left\{Z_{N}: x_{\imath}=x_{\jmath},\left|v_{\imath}-v_{\jmath}\right|=3,\right\} .
$$

Taking now the expectation of Eq. (3.11) we obtain the searched equation for $\mu^{N}$ :

$$
\begin{aligned}
& \frac{d}{d t} \int \mathrm{d} Z_{N} \Phi\left(Z_{N}\right) \mu^{N}\left(Z_{N}, t\right)=\int \mathrm{d} Z_{N} \sum_{i=1}^{N} v_{\imath} \partial_{i} \Phi\left(Z_{N}\right) \mu^{N}\left(Z_{N}, t\right) \\
& +\frac{3}{2} \sum_{i \neq j} \varepsilon \int_{\mathscr{F}_{\imath \jmath}} \mathrm{d} Y \Phi(Y)\left\{\mu^{N}\left(Y^{\prime}(i, j), t\right)-\mu^{N}(Y, t)\right\} .
\end{aligned}
$$

The last passage is justified by our Definition (3.5) of the flow on $\mathscr{F}^{\omega}$ : indeed the collision between the pair $(i, j)$ is not included in the random variable in curl brackets in (3.11) (the so-called collision operator) which turns out to be independent of $\omega(i, j)$. Notice that the other possible choice of representing the elements of $\mathscr{F}^{\omega}$ in terms of outgoing velocities would create problems in taking the expectation in (3.11) and moreover would give the opposite sign to the collision operator.

Let us now derive the first equation (for $j=1$ ) of the hierarchy (2.9). We consider in (3.12) a test function $\Phi=\Phi\left(z_{1}\right)$ depending only on the first variable and using the symmetry property of $\mu^{N}$ we obtain:

$$
\begin{aligned}
& \frac{d}{d t} \int \mathrm{d} z_{1} \Phi\left(z_{1}\right) f_{1}^{N}\left(z_{1}, t\right)=\int \mathrm{d} z_{1}\left(v_{1} \partial_{1} \Phi\right)\left(z_{1}\right) f_{1}^{N}\left(z_{1}, t\right) \\
& \quad+3(N-1) \varepsilon \int \mathrm{d} z_{1} \Phi\left(z_{1}\right)\left\{f_{2}^{N}\left(x_{1}, v_{1}^{\prime}, x_{1}, v_{2}^{\prime} ; t\right)-f_{2}^{N}\left(x_{1}, v_{1}, x_{1}, v_{2} ; t\right)\right\}
\end{aligned}
$$

with $\left|v_{2}-v_{1}\right|=3$. 

and

Furthermore it is not hard to see that $f_{1}^{N}$ is differentiable along the stream flow

$$
\begin{aligned}
\frac{d}{d t} f_{1}^{N}\left(x_{1}+v_{1} t, v_{1} ; t\right)= & 3(N-1) \varepsilon\left\{f_{2}^{N}\left(x_{1}+v_{1} t, v_{1}^{\prime}, x_{1}+v_{1} t, v_{2}^{\prime} ; t\right)\right. \\
& \left.-f_{2}^{N}\left(x_{1}+v_{1} t, v_{1}, x_{1}+v_{1} t, v_{2} ; t\right)\right\}
\end{aligned}
$$

We have now given a rigorous meaning (actually we have derived the first equation of the hierarchy (2.9) only, but this is what we need) to Eqs. (2.7) and (2.9), so that we are ready to prove two basic estimates which will be useful in Sect. 5 .

From now on we will assume $\omega$ fixed. We recall that $f_{j}^{N}\left(Z_{j}, t \mid \omega\right)$, the marginal distribution densities of $\mu_{\omega}^{N}$, are defined in analogy to (2.8); moreover we will sometimes be concerned with the "trace" of $f_{2}^{N}(\cdot \mid \omega)$ defined as

$$
f_{2}^{N}\left(x_{1}, v_{1}, x_{1}, v_{2} ; t \mid \omega\right)=\int \mathrm{d} Z_{N-2} \mu_{0}^{N}\left(T_{\omega}^{-t}\left(x_{1}, v_{1}, x_{1}, v_{2}, Z_{N-2}\right)\right) .
$$

Finally, all through the paper the measure $\mu_{0}^{N}$ will be assumed as factorizing, that is

$$
\mu_{0}^{N}\left(Z_{N}\right)=\prod_{i=1}^{N} f_{0}\left(z_{\imath}\right)
$$

with

$$
\left\|f_{0}\right\|_{L_{\infty}} \leq A \text {. }
$$

Denote by $k$ the number of (possible) collisions among the $N$ particles, that is

$$
k(\omega)=\sum_{i \neq j=1}^{N} \omega(i, j)
$$

then we have the following result:

Proposition 3.1. Let $\omega(1,2)=1$ and $\left|v_{2}-v_{1}\right|=3$. Then:

$$
\int_{0}^{\infty} \mathrm{d} t \int \mathrm{d} x_{1} f_{2}^{N}\left(x_{1}, v_{1}, x_{1}, v_{2} ; t \mid \omega\right) \leq \frac{k(\omega)}{3} .
$$

Proof.

$$
\begin{aligned}
& \int_{0}^{\infty} \mathrm{d} t \quad \int_{v_{3} \ldots v_{N}} \mathrm{~d} x_{1} f_{2}^{N}\left(x_{1}, v_{1}, x_{1}, v_{2}, t \mid \omega\right)=\sum_{0}^{\infty} \mathrm{d} t \\
& \quad \times \int \mathrm{d} x_{1} \mathrm{~d} x_{3} \ldots \mathrm{d} x_{N} \mu_{0}^{N}\left(T_{\omega}^{-t}\left(x_{1}, v_{1}, x_{1}, v_{2}, \ldots, x_{N} v_{N}\right)\right) \chi(1,2) \\
& \leq \int_{0}^{\infty} \mathrm{d} t \int_{\mathscr{F}_{1,2}^{\omega}} \mathrm{d} Y \mu_{0}^{N}\left(T_{\omega}^{-t}(Y)\right) \leq \int_{0}^{\infty} \mathrm{d} t \int_{\mathscr{\mathcal { F }} \omega} \mathrm{d} Y \mu_{0}^{N}\left(T_{\omega}^{-t}(Y)\right) .
\end{aligned}
$$

Define

$$
\begin{aligned}
\beta(Y) & =\inf \left\{\tau>0: S^{-\tau} Y \in \mathscr{F}^{\omega}\right\} \\
& =\infty \text { if } S^{-t} Y \notin \mathscr{F}^{\omega} \text { for all } t \in(0, \infty)
\end{aligned}
$$


then the mapping $R: \mathscr{F}^{\omega} \rightarrow \mathscr{F}^{\omega}$

$$
R(Y)=\pi\left(S^{-\beta(Y)} Y\right)
$$

is invertible and preserves the measure $d Y$. Now we have

$$
\begin{aligned}
\int_{0}^{\infty} \mathrm{d} t \int_{\mathscr{F} \omega} \mathrm{d} Y \mu_{0}^{N}\left(T_{\omega}^{-t} Y\right)= & \sum_{r=0}^{k(\omega)-1} \sum_{l=0}^{r} \int_{\mathscr{F} \omega} \mathrm{d} Y \chi\left(A_{r}\right) \\
& \times \int_{0}^{\beta\left(R^{l}(Y)\right)} \mathrm{d} t \mu_{0}^{N}\left(S^{-t} R^{l}(Y)\right),
\end{aligned}
$$

where

$$
A_{r}=\left\{Y \in \mathscr{F}^{\omega}: T_{\omega}^{-t} Y \text { displays } r \text { collisions if } t \in(0, \infty)\right\},
$$

and from now on $\chi(A)$ will denote the characteristic function of the set $A$. Notice that if $Y \in A_{r}$ then $\beta\left(R^{r}(Y)\right)=+\infty$.

Since $R$ is measure preserving, we have:

$$
\begin{aligned}
(3.21) & \leq \sum_{l=0}^{k(\omega)-1} \int_{\mathscr{F} \omega} \mathrm{d} Y \int_{0}^{\beta\left(R^{l}(Y)\right)} \mathrm{d} t \mu_{0}^{N}\left(S^{-t} R^{l}(Y)\right) \\
& \leq k(\omega) \int_{\mathscr{T} \omega} \mathrm{d} Y \int_{0}^{\beta(Y)} \mathrm{d} t \mu_{0}^{N}\left(S^{-t} Y\right) .
\end{aligned}
$$

Notice that $Y$ is of the form $\left(x_{1}, v_{1}, \ldots x_{\imath} v_{i} \ldots x_{\imath} v_{j} \ldots x_{N} v_{N}\right)$ for a couple $(i, j)$, so that by (3.22) we get

$$
\begin{aligned}
(3.21) \leq & k(\omega) \int_{\mathscr{T} \omega} \mathrm{d} Y \int_{0}^{\infty} \mathrm{d} t \mu_{0}^{N}\left(S^{-t} Y\right) \\
= & k(\omega) \int_{\mathscr{T} \omega} \mathrm{d} Y \int_{0}^{\infty} \mathrm{d} t \mu_{0}^{N}\left(x_{1}-v_{1} t, v_{1} \ldots x_{\imath}-v_{i} t, v_{\imath} \ldots x_{i}\right. \\
& \left.-v_{\jmath} t, v_{j} \ldots x_{N}-v_{N} t, v_{N}\right) \\
= & k(\omega) \int_{\mathscr{T} \omega} \mathrm{d} Y \int_{0}^{\infty} \mathrm{d} t \mu_{0}^{N}\left(x_{1}, v_{1}, \ldots x_{i}, v_{i} \ldots x_{i}-\left(v_{j}-v_{i}\right) t, v_{j} \ldots x_{N}, v_{N}\right) \\
\leq & \frac{k(\omega)}{3} \int_{0} \mathrm{~d} Z_{N} \mu_{0}^{N}\left(Z_{N}\right)=\frac{k(\omega)}{3},
\end{aligned}
$$

from which we complete the proof.

Our next goal in this section is to give an estimate on the probability density of one particle $f_{1}^{N}(\cdot \mid \omega)$. To this purpose we need some more definitions which will play an important role in Sect. 5.

Given an integer $k$, we denote by $I_{k}=\{1,2, \ldots k\}$ the set of the first $k$ integers, while $I$ will stand for any subset of indices of $I_{N}$. 
Given $\omega \in \Omega$, we denote by $\operatorname{ch}(i)$ ("chain" starting from $i$ ) any set $I \subset I_{N}$ of indices such that

$$
\omega\left(i, i_{1}\right) \omega\left(i_{1}, i_{2}\right) \ldots \omega\left(i_{k-1}, i_{k}\right)=1
$$

for some ordering $i_{1}, \ldots, i_{k}$ of the set $I$. The union of all chains starting from the particle $i$ will be called "cluster of $i$ " and denoted by

$$
\operatorname{cl}(i)=\bigcup \operatorname{ch}(i)
$$

Notice that the index $i$ is not included in $\operatorname{cl}(i)$. Finally we denote by

$$
n(I):=\sum_{\substack{h \neq k \\ h, k \in I}} \omega(h, k)
$$

the number of collisions occurring in $I$. We want to stress that

$$
|\operatorname{cl}(i)| \leq n(\operatorname{cl}(i) \cup\{i\}) \leq \frac{N(N-1)}{2}
$$

(here and after $|A|$ stands for the cardinality of the set $A$ ) so that $|\operatorname{cl}(i)|$ is the minimum number of collisions in order to set the cluster.

Proposition 3.2. Given $\omega \in \Omega$, assume that

$$
n(\operatorname{cl}(1) \cup\{1\})=|\operatorname{cl}(1)|=h .
$$

Then

$$
f_{1}^{N}\left(x_{1}, v_{1} ; t \mid \omega\right)=\int \mathrm{d} Z_{N-1} \mu_{\omega}^{N}\left(Z_{N}, t\right) \leq 4 A\left(\frac{3}{2}\right)^{h}
$$

Proof. Let $\varphi=\varphi\left(z_{1}\right)$ be a positive real function such that

$$
\int \mathrm{d} z_{1} \varphi\left(z_{1}\right)=1
$$

Then we have:

$$
\int \mathrm{d} z_{1} f_{1}^{N}\left(z_{1} ; t \mid \omega\right) \varphi\left(z_{1}\right)=\int \mathrm{d} Z_{N} \mu_{0}^{N}\left(Z_{N}\right) \varphi\left(\left(T_{\omega}^{t} Z_{N}\right)_{1}\right)
$$

where $\left(T_{\omega}^{t} Z_{N}\right)_{1}$ denotes position and velocity of the first particle of the configuration $T_{\omega}^{t} Z_{N}$.

Let us put $\left(T_{\omega}^{t} Z_{N}\right)_{1}=(y, u)$ and consider, for fixed $v_{1}, z_{2}, \ldots, z_{N}$, the mapping

$$
x_{1} \mapsto y
$$

We will show that

$$
\frac{\partial y}{\partial x_{1}} \geq\left(\frac{2}{3}\right)^{h}
$$


which implies that the mapping (3.27) is invertible, so that we can conclude, by (3.26) and (3.16) and (3.17):

$$
\begin{gathered}
\int \mathrm{d} z_{1} f_{1}^{N}\left(z_{1} ; t \mid \omega\right) \varphi\left(z_{1}\right) \leq A \int \mathrm{d} Z_{N-1} \mu_{0}^{N}\left(Z_{N-1}\right) \int \mathrm{d} z_{1} \varphi(y, u) \\
\leq A\left(\frac{3}{2}\right)^{h} \int \mathrm{d} Z_{N-1} \mu_{0}^{N}\left(Z_{N-1}\right) \sum_{v_{1}} \int \mathrm{d} y \varphi\left(y, u\left(y, v_{1}, Z_{N-1}\right)\right) \\
\leq 4 A\left(\frac{3}{2}\right)^{h} \int \mathrm{d} Z_{N-1} \mu_{0}^{N}\left(Z_{N-1}\right) \sum_{u} \int \mathrm{d} y \varphi(y, u)=4 A\left(\frac{3}{2}\right)^{h}
\end{gathered}
$$

which is our thesis.

Thus, the whole proof relies upon estimate (3.28), which we now prove. Let us start by analyzing $T_{\omega}^{t} Z_{N}$. Let us denote by $0<t_{1}<t_{2}<\ldots<t_{m}<t$ the collision times and by $\left(i_{j}, s_{\jmath}\right)$ the pair of particles colliding at time $t_{\jmath}, j=1, \ldots, m$. Putting

$$
b_{j}=\frac{1}{3}\left(v_{i_{j}}+v_{s_{j}}\right)
$$

we have, for $\tau \in\left(t_{1}, t_{2}\right)$ :

$$
\begin{aligned}
& x_{i_{1}}(\tau)=\left(1-b_{1}\right) x_{\imath_{1}}+b_{1} x_{s_{1}}-v_{s_{1}} \tau, \\
& x_{s_{1}}(\tau)=-b_{1} x_{\iota_{1}}+\left(1+b_{1}\right) x_{s_{1}}-v_{\iota_{1}} \tau,
\end{aligned}
$$

since $v_{i_{1}}^{\prime}=-v_{s_{1}}$ and $v_{s_{1}}^{\prime}=-v_{i_{1}}$. Hence,

$$
T_{\omega}^{r} Z_{N}=\left(X_{N}(\tau), V_{N}(\tau)\right)=\left(W_{1} X_{N}+V_{N}^{\prime}(1) \tau, V_{N}^{\prime}(1)\right),
$$

where $V_{N}^{\prime}(1)=\left(v_{1}, \ldots, v_{i_{1}}^{\prime}, \ldots, v_{s_{1}}^{\prime}, \ldots v_{N}\right)$ and $W_{1}$ is the $N \times N$ matrix whose elements $\left(W_{1}\right)_{r, l}$ are the following:

$$
\begin{aligned}
& \left(W_{1}\right)_{r, r}= \begin{cases}1 & \text { if } r \neq i_{1} \text { or } s_{1} \\
1-b_{1} & \text { if } r=i_{1} \\
1+b_{1} & \text { if } r=s_{1}\end{cases} \\
& \left(W_{1}\right)_{r, l}= \begin{cases}b_{1} & \text { if }(r, l)=\left(i_{1}, s_{1}\right) \\
-b_{1} & \text { if }(r, l)=\left(s_{1}, i_{1}\right) \\
0 & \text { otherwise }\end{cases}
\end{aligned}
$$

By iterating $m$ times formula (3.32) we have at time $t$ :

$$
\begin{aligned}
X_{N}(t)= & \left(x_{1}(t), \ldots, x_{N}(t)\right)=W_{m} W_{m-1} \ldots W_{1} X_{N} \\
& +\sum_{r=1}^{m-1} W_{m} W_{m-1} \ldots W_{r+1} V_{N}^{\prime}(r)\left(t_{r}-t_{r-1}\right)+V_{N}^{\prime}\left(t_{m}\right)\left(t-t_{m}\right),
\end{aligned}
$$

where $W_{\jmath}, j=1, \ldots, m$, are the analogues of $W_{1}$, associated to the $j$-th collision and are defined as in (3.33) replacing the index 1 by $j$ everywhere. Similarly,

$$
V_{N}^{\prime}(r)=\left(v_{1}, \ldots, v_{i_{r}}^{\prime}, \ldots, v_{s_{r}}^{\prime}, \ldots, v_{N}\right) .
$$

Putting

$$
W^{(m)}=W_{m} \ldots W_{1},
$$


we easily realize that

$$
\frac{\partial y}{\partial x_{1}}=\left(W^{(m)}\right)_{1,1}
$$

so that to prove (3.28) we have to give more details on the matrix $W^{(m)}$.

Since we have, by definition:

$$
\left(W^{(m)}\right)_{1,1}=\left(W_{m} W^{(m-1)}\right)_{1,1}=\sum_{r=1}^{N}\left(W_{m}\right)_{1, r}\left(W^{(m-1)}\right)_{r, 1}
$$

there are two possibilities:

(i) the particle 1 is not involved in the $m$-th collision. In this case

$$
\left(W^{(m)}\right)_{1,1}=\left(W^{(m-1)}\right)_{1,1},
$$

(ii) the particle 1 collides with particle $s_{m}$. Then

$$
\left(W^{(m)}\right)_{1,1}=\left(1-b_{m}\right)\left(W^{(m-1)}\right)_{1,1}+b_{m}\left(W^{(m-1)}\right)_{s_{m}, 1} .
$$

We are going to show that in case (ii), under our assumptions, we have

$$
\left(W^{(m-1)}\right)_{s_{m}, 1}=0 \text {. }
$$

Indeed:

$$
\left(W^{(m-1)}\right)_{s_{m}, 1}=\sum_{r=1}^{N}\left(W_{m-1}\right)_{s_{m}, r}\left(W^{(m-2)}\right)_{r, 1},
$$

and iterating this formula we obtain

$$
\left(W^{(m-1)}\right)_{s_{m}, 1}=\sum_{l} \alpha_{l}\left(W_{1}\right)_{l, 1}
$$

where $\alpha_{l}$ are suitable coefficients and the sum is taken over the indices $l$ of particles $\sigma_{1}, \ldots, \sigma_{p}$, each of them being linked to particle 1 by a chain. An element $\left(W_{1}\right)_{l, 1}$ is different from zero only if there is a collision between the particle 1 and one of the $\sigma_{\imath}$ 's, $i=1, \ldots, p$, but this is not allowed by our hypothesis since a collision of this kind would necessarily imply $n(\operatorname{cl}(1))>|\operatorname{cl}(1)|$. Thus (3.40) is proven and we can conclude by (3.39):

$$
\left(W^{(m)}\right)_{1,1} \geq \frac{2}{3}\left(W^{(m-1)}\right)_{1,1}
$$

which, by iteration, implies the thesis by (3.37).

\section{The Main Result and the Strategy of the Proof}

From now on $c$ will denote any positive constant independent of $N$ and $t$. The main result in this paper is the following theorem.

Theorem 4.1. Suppose $f_{0} \in L_{\infty}$ is an initial datum for the Boltzmann equation (2.14) and $f=f(x, v, t)$ is the solution of the initial value problem. Let $\left\{f_{j}^{N}\right\}$ be the $j$-particle distributions associated to the measure $\mu^{N}\left(Z_{N}, t\right)$, solution to Eq. (2.7) with 
initial condition $\mu_{0}^{N}\left(Z_{N}\right)=\prod_{i=1}^{N} f_{0}\left(z_{i}\right)$. Then, there exists $\lambda_{0}>0$ (independent of $t$ and $f_{0}$ ) such that for $\lambda=N \varepsilon<\lambda_{0}$, for all $j$ and $t \geq 0$, the following holds:

$$
\lim _{N \rightarrow \infty} \int\left|f_{\jmath}^{N}\left(Z_{j}, t\right)-\prod_{i=1}^{\jmath} f\left(z_{i}, t\right)\right| \mathrm{d} Z_{j}=0 .
$$

The proof of the above theorem follows from the following fundamental proposition, which we shall illustrate in this section and prove in the next one.

Proposition 4.1. Under the same assumptions as in Theorem 4.1, there exist a function $\tilde{f}^{N}: \mathbf{R} \times V \rightarrow \mathbf{R}^{+}$and a sequence of functions $g_{\jmath}^{N}: \mathbf{R}^{j} \times V^{j} \rightarrow \mathbf{R}^{+}, j=1, \ldots, N$ such that the following holds:

$$
f_{j}^{N}\left(z_{1}, \ldots, z_{j} ; t\right)=\prod_{k=1}^{\jmath} \tilde{f}^{N}\left(z_{k}, t\right)+g_{j}^{N}\left(z_{1}, \ldots, z_{j} ; t\right)
$$

with the properties

$$
\begin{gathered}
\tilde{f}^{N} \leq c \quad(c \text { independent of } t \text { and } N), \\
\left\|g_{j}^{N}\right\|_{L_{1}} \leq \frac{c^{\jmath}}{N}, \\
\sum_{\substack{v_{1}, v_{2} \\
\left|v_{1}-v_{2}\right|=3}} \int_{0}^{\infty} \mathrm{d} t \int \mathrm{d} x\left|g_{2}^{N}\left(x, v_{1}, x, v_{2}\right)\right| \leq \frac{c}{N} .
\end{gathered}
$$

Proof of Theorem 4.1. By (2.9), (2.14) and (4.2),

$$
\begin{aligned}
\left\|f(t)-f_{1}^{N}(t)\right\|_{L_{1}} \leq & 6(\lambda-\varepsilon) \int_{0}^{t} \mathrm{~d} \tau \\
& \times \int \mathrm{d} x \sum_{\substack{v, v_{1} \\
\left|v-v_{1}\right|=3}} f(x, v ; \tau)\left|f\left(x, v_{1} ; \tau\right)-\tilde{f}^{N}\left(x, v_{1} ; \tau\right)\right| \\
& \left.+\tilde{f}^{N}\left(x, v_{1} ; \tau\right)\left|f(x, v ; \tau)-\tilde{f}^{N}(x, v ; \tau)\right|\right\} \\
& +6 \varepsilon \int_{0}^{t} \mathrm{~d} \tau \int_{0} \mathrm{~d} x \sum_{v, v_{1}} f(x, v ; \tau) f\left(x, v_{1} ; \tau\right) \\
& +6(\lambda-\varepsilon) \int_{0}^{t} \mathrm{~d} \tau \int_{\substack{\left|v-v_{1}\right|=3 \\
\mathrm{~d} x}}\left|\sum_{v, v_{1}}\right| g_{2}^{N}\left(x, v, x, v_{1} ; \tau\right) \mid
\end{aligned}
$$

the factor 6 in (4.6) coming from the trivial observation that $\sum_{v, v_{1}}=\sum_{v^{\prime}, v_{1}^{\prime}}$. 
Since $f$ is bounded in $L_{\infty}$ (see [4]) by (4.3), (4.4) and (4.5) we obtain for $t \leq T$ ( $T$ arbitrarily fixed):

$$
\begin{aligned}
\left\|f(t)-f_{1}^{N}(t)\right\|_{L_{1}} & \leq \frac{c(1+T)}{N}+c \int_{0}^{t} \mathrm{~d} \tau\left\|f(\tau)-\tilde{f}^{N}(\tau)\right\|_{L_{1}} \\
& \leq \frac{c(1+T)}{N}+c \int_{0}^{t} \mathrm{~d} \tau\left\|f(\tau)-f_{1}^{N}(\tau)\right\|_{L_{1}}+c \int_{0}^{T} \mathrm{~d} \tau\left\|g_{1}^{N}(\tau)\right\|_{L_{1}} \\
& \leq \frac{c(1+T)}{N}+c \int_{0}^{t} \mathrm{~d} \tau\left\|f(\tau)-f_{1}^{N}(\tau)\right\|_{L_{1}}
\end{aligned}
$$

so that for any $t \in[0, T]$ we have by the Gronwall's Lemma,

$$
\left\|f(t)-f_{1}^{N}(t)\right\|_{L_{1}} \rightarrow 0
$$

Finally, since by (4.4) it is also

$$
\left\|f(t)-\tilde{f}^{N}(t)\right\|_{L_{1}} \rightarrow 0
$$

we can conclude the proof.

Remark. In the proof of Theorem 4.1 we have made use of the fact that there exists a $L_{\infty}$-solution to the Boltzmann equation 2.14. However this is not a necessary assumption, since proceeding as in this proof we could show that the sequence $\left\{f_{1}^{N}\right\}$ is a Cauchy sequence in $L_{1}$ and its limit satisfies Eq. (2.14).

Let us now argue about the meaning and the implications of Proposition 4.1. We know, following Lanford's arguments [1], that an estimate of the $j$-particle distributions of the kind $\left\|f_{j}^{N}\right\|_{L_{\infty}}<c^{j}$ would imply the convergence, but it seems very hard to deduce it for a large time interval, even for $\lambda$ small, starting directly from the hierarchy of Eqs. (2.9). Thus we change point of view. The leading idea in this paper is to show that the dynamics of our $N$-particle system can be interpreted as a "cluster dynamics" in the following sense: for $\lambda$ small the cluster of any particle $i$ is, with large probability, minimal (i.e. $n(\operatorname{cl}(i))=|\operatorname{cl}(i)|$ ) and this implies that any tagged group of particles have non-overlapping clusters. Moreover, the probability of having $|\mathrm{cl}(i)|$ large is exponentially small and this fact, together with the previous considerations allow us to say that the $f_{\jmath}^{N}$ are to be interpreted as "almost" factorizing, but for a small (order $1 / N$ ) error.

\section{Proof of Proposition 4.1}

For any subset $I$ of the integers we put

$$
Z_{I}=\left\{z_{\imath}\right\}_{\imath \in I}
$$


We have, by definition:

$$
\begin{aligned}
f_{\jmath}^{N}\left(Z_{\jmath} ; t\right)= & \mathbf{E}\left[\int \mathrm{d} Z_{N-\jmath} \mu_{0}^{N}\left(T_{\omega}^{-t} Z_{N}\right)\right] \\
= & \sum_{h \geq 0} \sum_{\substack{I \subset I_{j}^{c} \\
|I|=h}} \sum_{k \geq h} \mathbf{E}\left[\chi\left(\operatorname{cl}\left(I_{j}\right)=I\right) \chi\left(n\left(I \cup I_{j}\right)=k\right)\right. \\
& \left.\times \int \mathrm{d} Z_{N-\jmath} \mu_{0}^{N}\left(T_{\omega}^{-t} Z_{N}\right)\right]
\end{aligned}
$$

where, as before, $n\left(I \cup I_{\jmath}\right)=\sum_{\imath, k \in I \cup I_{\jmath}} \omega(i, k)$ and $\operatorname{cl}(I)=\bigcup_{i \in J} \operatorname{cl}(i) / I$.

Let us decompose $f_{j}^{N}$ as:

$$
f_{j}^{N}=\bar{f}_{\jmath}^{N}+R_{j}^{N}
$$

where $\bar{f}_{j}^{N}$ represents the contribution to $f_{j}^{N}$ by those $\omega$ 's for which $n\left(I \cup I_{j}\right)$ is minimal that is:

$$
\begin{aligned}
\bar{f}_{j}^{N}\left(Z_{\jmath} ; t\right)= & \sum_{h \geq 0} \sum_{\substack{I \subset I_{j}^{c} \\
|I|=h}} \mathbf{E}\left[\chi\left(\operatorname{cl}\left(I_{j}\right)=I\right) \chi\left(n\left(I \cup I_{\jmath}\right)=h\right)\right. \\
& \left.\times \int \mathrm{d} Z_{N-j} \mu_{0}^{N}\left(T_{\omega}^{-t} Z_{N}\right)\right],
\end{aligned}
$$

while $R_{j}^{N}$ is defined accordingly.

The fact that $n\left(I \cup I_{j}\right)=h$ necessarily implies that $I$ has to be the disjoint union of sets, i.e. $I=\bigcup_{s=1}^{j} J_{s}$, with $\left|J_{s}\right|=h_{s}$ and $\sum_{s=1}^{J} h_{s}=h$. Therefore:

$$
\begin{aligned}
\bar{f}_{j}^{N}\left(Z_{j} ; t\right)= & \sum_{h_{1}, \ldots, h_{\jmath}} \sum_{\substack{J_{1}, \ldots, J_{j} \\
\left|J_{s}\right|=h_{s} \\
J_{r} \cap J_{s}=0, r \neq s}} \mathbf{E}\left[\prod_{s=1}^{j} \chi\left(\operatorname{cl}(s)=J_{s}\right), \chi\left(n\left(\bar{J}_{s}\right)=h_{s}\right)\right. \\
& \left.\times \int \mathrm{d} Z_{J_{s}} \mu_{0}^{h_{s}+1}\left(T_{\omega}^{-t} Z_{\bar{J}_{s}}\right)\right],
\end{aligned}
$$

where $\bar{J}_{s}=J_{s} \cup\{s\}$.

Indeed:

$$
\begin{aligned}
\int \mathrm{d} Z_{N-j} \mu_{0}^{N}\left(T_{\omega}^{-t} Z_{N}\right) & =\int \mathrm{d} Z_{I} \mu_{0}^{h+j}\left(T_{\omega}^{-t} Z_{I \cup I_{\jmath}}\right) \\
& =\prod_{s=1}^{j} \int \mathrm{d} Z_{J_{s}} \mu_{0}^{h_{s}+1}\left(T_{\omega}^{-t} Z_{\bar{J}_{s}}\right)
\end{aligned}
$$

due to the definition of cluster and to the fact that clusters of different particles have no intersection. 

define:

We will show that $\bar{f}_{j}^{N}$ is "almost" a product of functions. More precisely let us

$$
\begin{aligned}
\tilde{f}^{N}\left(z_{s} ; t\right)= & \sum_{h \geq 0} \sum_{J_{s}:\left|J_{s}\right|=h} \mathbf{E}\left[\chi\left(\operatorname{cl}(s)=J_{s}\right) \chi\left(n\left(\bar{J}_{s}\right)=h\right)\right. \\
& \left.\times \int \mathrm{d} Z_{J_{s}} \mu_{0}^{h+1}\left(T_{\omega}^{-t} Z_{\tilde{J}_{s}}\right)\right]
\end{aligned}
$$

and

$$
h_{j}^{N}\left(Z_{\jmath} ; t\right)=\bar{f}_{\jmath}^{N}\left(Z_{\jmath} ; t\right)-\prod_{s=1}^{j} \tilde{f}^{N}\left(z_{s} ; t\right) .
$$

Before proving that $h_{?}^{N}$ is small in $L_{1}$ we need to get a $L_{\infty}$-estimate of $\tilde{f}^{N}$. To this purpose we note that by Proposition 3.2:

$$
\begin{aligned}
\tilde{f}\left(z_{1}, t\right) & \leq \sum_{h \geq 0} \sum_{I:|I|=h}\left(\frac{3}{2}\right)^{h+1} \mathbf{E}[\chi(\operatorname{cl}(1)=I) \chi(n(\bar{I})=h)] \\
& \leq \sum_{h \geq 0} \sum_{I:|I|=h}\left(\frac{3}{2}\right)^{h+1} \mathbf{E}[\chi(\operatorname{cl}(1)=I)]
\end{aligned}
$$

Now, to estimate the above expectation we need to introduce one more definition. Given the set $I=\operatorname{cl}(1)$ with $|I|=h$, we call a "graph" of $I$ [denoted by $G(I)]$ a way to settle $h$ links among the $h+1$ particles of $\bar{I}$, in such a way that each particle is connected to the first through a chain.

Fig. 1.

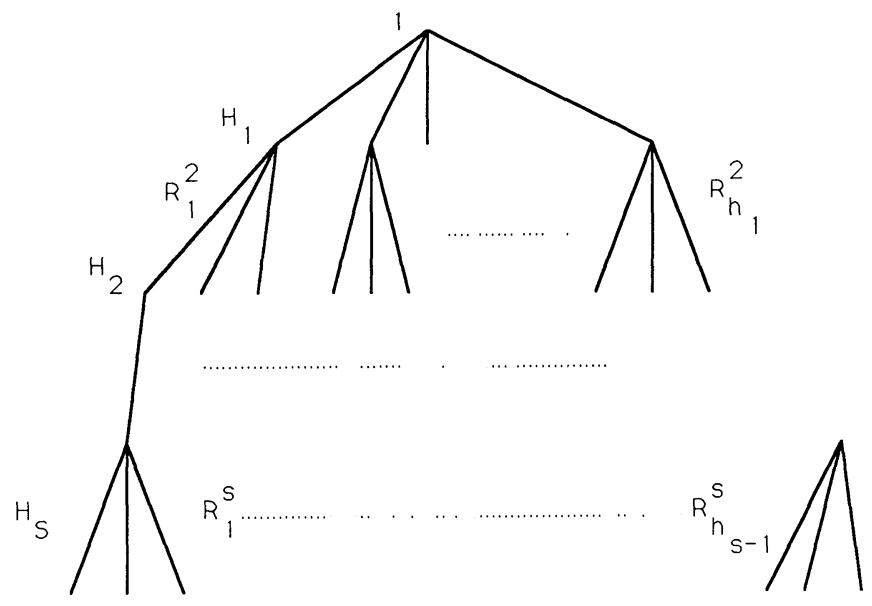

A graph is specified when a number $s \leq h$ is given, together with $s$ groups of particles $H_{1}, \ldots, H_{s}$ such that $\left|H_{\imath}\right|=h_{\imath}$ and arranged as follows: $H_{1}$ is connected with particle $1, H_{2}$ with particles of $H_{1}$ and so on up to $s$, so that $\sum_{i=1}^{s} h_{i}=h$. Moreover $H_{\imath}=\bigcup_{l=1}^{h_{\imath-1}} R_{l}^{i}$, where $R_{l}^{\imath}$ is the group of particles of $H_{i}$ which is connected with the 
$l$-th particle of the group $H_{\imath-1},\left|R_{l}^{\imath}\right|=r_{l}^{\imath}$ and $\sum_{l=1}^{h_{\imath-1}} r_{l}^{i}=h_{i}$. Notice that $h_{\imath}$ is always different from zero, while $r_{l}^{i}$ may possibly be zero.

Given a graph $G(I)$ we denote by $\chi(G(I))$ the characteristic function of the event " $G(I)$ is realized," that is $\omega(i, j)=1$ if $i$ and $j$ are connected by a link in the graph. We want to stress the fact that if $\operatorname{cl}(1)=I$, then at least a graph $G(I)$ is realized. Therefore:

$$
\chi(\operatorname{cl}(1)=I) \leq \sum_{G(I)} \chi(G(I))
$$

which implies

$$
\mathbf{E}(\chi(\operatorname{cl}(1))=I) \leq \sum_{G(I)} \varepsilon^{h}
$$

Moreover

$$
\begin{aligned}
& \sum_{G(I)} 1=\sum_{s=1}^{h} \sum_{\substack{h_{1}, \ldots, h_{s} \\
h_{2}>0 \\
\sum h_{2}=h}}\left(\begin{array}{c}
h \\
h_{1}
\end{array}\right)\left(\begin{array}{c}
h-h_{1} \\
h_{2}
\end{array}\right) \ldots\left(\begin{array}{c}
h-\sum_{i=1}^{s-1} h_{i} \\
h_{s}
\end{array}\right) \\
& \times \prod_{\substack { l=2 \\
\begin{subarray}{c}{r_{1}^{l} \ldots r_{h_{l-1}}^{l} \\
\sum r_{\imath}^{l}=h_{l}{ l = 2 \\
\begin{subarray} { c } { r _ { 1 } ^ { l } \ldots r _ { h _ { l - 1 } } ^ { l } \\
\sum r _ { \imath } ^ { l } = h _ { l } } }\end{subarray}}\left(\begin{array}{c}
h_{l} \\
r_{1}^{l}
\end{array}\right)\left(\begin{array}{c}
h_{l}-r_{1}^{l} \\
r_{2}^{l}
\end{array}\right) \ldots\left(\begin{array}{c}
h_{l}-\sum_{i=1}^{h_{l-1}-1} r_{i}^{l} \\
r_{h_{l}-1}^{l}
\end{array}\right) \\
& =\sum_{s=1}^{h} \sum_{\substack{h_{1}, \ldots, h_{s} \\
h_{2}>0 \\
\sum h_{2}=h}} \frac{h !}{h_{1} ! \ldots h_{s} !} \prod_{\substack{l=2 \\
r_{1}^{l} \ldots r_{h_{l-1}}^{l} \\
\sum r_{l}^{l}=h_{l}}}^{s} \frac{h_{l} !}{r_{1}^{l} ! \ldots r_{h_{l-1}}^{l} !} \\
& \leq \sum_{s=1}^{h} \sum_{\substack{h_{1}, \ldots, h_{s} \\
h_{2}>0 \\
\sum h_{\imath}=h}} h ! e^{h} \leq h ! c^{h}
\end{aligned}
$$

The last passage in (5.12) follows from the fact that:

$$
\sum_{\substack{h_{1} \ldots h_{s} \\ \sum h_{\imath}=h}} 1=\sum_{\substack{h_{1} \ldots h_{s} \\ \sum h_{i}=h}} e^{h_{i}} e^{-h_{i}} \leq e^{h}\left(\sum_{h_{i}>0} e^{-h_{i}}\right)^{s}=e^{h}\left(\frac{e}{e-1}\right)^{s} .
$$

Hence (5.11) and (5.12) imply that

$$
\mathbf{E}\left(\chi(\operatorname{cl}(1)=I) \leq(\varepsilon c)^{h} h !,\right.
$$

which, inserted in (5.9) gives us:

$$
\tilde{f}^{N}\left(z_{1}, t\right) \leq \sum_{h \geq 0}\left(\begin{array}{l}
N \\
h
\end{array}\right)(\varepsilon c)^{h} h ! \leq \sum_{h \geq 0} \frac{N !}{(N-h) !}(c \lambda)^{h} \frac{1}{N^{h}} \leq \sum_{h \geq 0}(c \lambda)^{h} \leq c
$$

for $\lambda \leq \lambda_{0}$, a sufficiently small constant. Thus 4.3 is proven. 
Let us now go back to (5.3) and (5.8): to prove (4.4) in Proposition 4.1 we have to show that $g_{j}^{N}:=R_{j}^{N}+h_{j}^{N}$ is small in the $L_{1}$-norm. We start by estimating $R_{j}^{N}$. It is by definition:

$$
\begin{aligned}
R_{\jmath}^{N}\left(Z_{\jmath}, t\right)= & \sum_{h \geq 0} \sum_{\substack{I \subset I_{j}^{c} \\
|I|=h}} \sum_{k>h} \mathbf{E}\left[\chi\left(\operatorname{cl}\left(I_{\jmath}\right)\right)=I\right) \chi\left(n\left(I \cup I_{j}\right)=k\right) \\
& \left.\times \int \mathrm{d} Z_{N-\jmath} \mu_{0}^{N}\left(T_{\omega}^{-t} Z_{N}\right)\right] .
\end{aligned}
$$

Let $J_{1}, \ldots, J_{1}$ be defined as follows:

$$
\begin{aligned}
J_{1} & =\operatorname{cl}(1), \\
J_{k} & =\operatorname{cl}(k) / J_{k-1}, \quad k=2, \ldots, j .
\end{aligned}
$$

Then, by definition, the $J_{k}$ 's are disjoint "subclusters," $J_{k} \subseteq \operatorname{cl}(k)$, union of some chains starting from $k$.

Thus, by (5.15) (using the fact that $\int \mathrm{d} Z_{N} \mu_{0}^{N}\left(T_{\omega}^{-t} Z_{N}\right)=1$ ) we have:

$$
\begin{aligned}
& \left\|R_{j}^{N}\left(Z_{\jmath}, t\right)\right\|_{L_{1}} \leq \sum_{h \geq 0} \sum_{\substack{I \subset I_{j}^{c} \\
|I|=h}} \sum_{k>h} \sum_{\substack{h_{1}, \ldots, h_{\jmath} \\
\sum h_{\imath}=h}} \\
& \times \sum_{\substack{J_{1}, \ldots, J_{j} \\
\left|J_{r}\right|=h_{r} \\
\sum_{r=1} J_{r}=1 \\
J_{r} \cap J_{s}=\phi \forall r \neq s}} \mathbf{E}\left[\prod_{r=1}^{r} \chi\left(\operatorname{cl}(r) \supseteq J_{r}\right) \chi\left(n\left(I \cup I_{j}\right)=k\right)\right] .
\end{aligned}
$$

Now, since

$$
\chi\left(\operatorname{cl}(r) \supseteq J_{r}\right) \leq \sum_{G\left(J_{r}\right)} \chi\left(G\left(J_{r}\right)\right)
$$

we have

$$
\begin{aligned}
\mathbf{E}[ & \left.\prod_{r=1}^{\jmath} \chi\left(\operatorname{cl}(r) \supseteq J_{r}\right) \chi\left(n\left(I \cup I_{j}\right)=k\right)\right] \\
& \times \sum_{G\left(J_{1}\right) \ldots G\left(J_{\jmath}\right)} \mathbf{E}\left[\chi\left(G\left(J_{1}\right) \ldots \chi\left(G\left(J_{\jmath}\right)\right) \chi\left(n\left(I \cup I_{j}\right)=k\right)\right] .\right.
\end{aligned}
$$

Since in the last sum $j$ graphs are to be fixed, also $h_{1}, \ldots, h_{j}$ links are fixed, such that $\sum_{i=1}^{j} h_{\imath}=h$. It only remains to fix $k-h$ links, which can be settled in $\left(\begin{array}{c}\mathscr{N}^{\circ} \\ k-h\end{array}\right)$

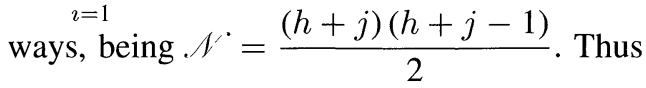

$$
(5.19) \leq \sum_{G\left(J_{1}\right) \ldots G\left(J_{j}\right)} \varepsilon^{k}\left(\begin{array}{c}
\mathscr{N} \\
k-h
\end{array}\right) \leq \prod_{r=1}^{j} h_{r} ! c^{h_{r}}\left(\begin{array}{c}
\mathscr{N} \\
k-h
\end{array}\right) \varepsilon^{k}
$$


by (5.12) and this implies:

$$
\begin{aligned}
\left\|R_{j}^{N}\left(Z_{j}, t\right)\right\|_{L_{1}} \leq & \sum_{h \geq 0} \sum_{k>h}\left(\begin{array}{c}
N \\
h
\end{array}\right) \sum_{\substack{h_{1}, \ldots, h_{j} \\
\sum h_{\imath}=h}} \frac{h !}{h_{1} ! \ldots h_{j} !} \\
& \times \prod_{r=1}^{j} h_{r} ! c^{h_{r}}\left(\begin{array}{c}
\mathscr{N}^{\cdot} \\
k-h
\end{array}\right) \varepsilon^{k} \\
\leq & \sum_{h \geq 0} \frac{N !}{(N-h) !} \sum_{h_{1}, \ldots, h_{j}}\left(\frac{c \lambda}{N}\right)^{h} \sum_{r=1}^{h}\left(\frac{\lambda}{N}\right)^{r}\left(\begin{array}{c}
\mathscr{N} \\
r
\end{array}\right) \\
\leq & \sum_{h \geq 0}(c \lambda)^{h} \sum_{r=1}^{. /}\left(\frac{\lambda}{N}\right)^{r}\left(\begin{array}{c}
\mathscr{N}^{\cdot} \\
r
\end{array}\right)
\end{aligned}
$$

since $k>h$. Recalling that

$$
\frac{\mathscr{N}}{N} \leq \frac{(h+j)^{2}}{N} \leq h+j
$$

we have:

$$
\begin{gathered}
\sum_{r=1}^{\prime \prime}\left(\frac{\lambda}{N}\right)^{r}\left(\begin{array}{c}
\mathscr{N}^{\circ} \\
r
\end{array}\right)=\left(1+\frac{\lambda}{N}\right)^{. \prime}-1 \leq \exp \left(\frac{\lambda \mathscr{N}^{\circ}}{N}\right)-1 \\
\leq \frac{\lambda \mathscr{N}^{\cdot}}{N} \exp \left(\frac{\lambda \mathscr{N}^{\circ}}{N}\right) \leq \lambda \frac{(h+j)^{2}}{N} \exp (\lambda(h+j))
\end{gathered}
$$

which, inserted in (5.21) gives us finally:

$$
\left\|R_{\jmath}^{N}\left(Z_{j}, t\right)\right\|_{L_{1}} \leq \frac{c^{j}}{N} \sum_{h \geq 0}(c \lambda)^{h} \leq \frac{c^{\jmath}}{N} .
$$

We will also need to control the term

$$
\int_{0}^{\infty} \mathrm{d} t \int \mathrm{d} x R_{2}^{N}\left(x, v_{1}, x, v_{2} ; t\right)
$$

in order to prove (4.5). This can be done by exactly performing the same procedure as before, with the only difference that, instead of bounding as in (5.17) the $L_{1}$-norm of

$$
\int \mathrm{d} Z_{N-2} \mu_{0}^{N}\left(T_{\omega}^{-t}\left(x, v_{1}, x, v_{2}, x_{3}, v_{3} \ldots x_{N}, v_{N}\right)\right)
$$

by one, we make use of Proposition 3.1. This implies that in all passages made from (5.17) up to (5.21) an extra divergent factor appears, which is $k \leq(h+j)^{2}$, but this does not affect the convergence of the geometric series, so that:

$$
\sum_{v_{1}} \int_{0}^{\infty} \mathrm{d} t \int \mathrm{d} x R_{2}^{N}\left(x, v_{1}, x, v_{2} ; t\right) \leq \frac{c}{N} .
$$


Now, let us estimate the $L_{1}$-norm of $h_{j}^{N}$ (see (5.8)). We have, by definition

$$
h_{j}^{N}=\mathscr{T}_{2}^{j}-\mathscr{T}_{1}^{j},
$$

where

$$
\begin{aligned}
\mathscr{T}_{1}^{j}= & \sum_{h_{1}, \ldots, h_{j}} \sum_{\substack{J_{1}, \ldots, J_{j} \\
\left|J_{i}\right|=h_{i}}}^{*} \prod_{r=1}^{j} \mathbf{E}\left[\gamma\left(z_{r}, h_{r}, J_{r} ; t\right)\right] \\
\mathscr{T}_{2}^{j}= & \sum_{h_{1}, \ldots, h_{j}} \sum_{\substack{J_{1}, \ldots, J_{j} \\
\left|J_{i}\right|=h_{i} \\
J_{r} \cap J_{s}=\phi, r \neq s}}\left\{\mathbf{E}\left[\prod_{r=1}^{j} \gamma\left(z_{r}, h_{r}, J_{r} ; t\right)\right]\right. \\
& \left.-\prod_{r=1}^{j} \mathbf{E}\left[\gamma\left(z_{r}, h_{r}, J_{r} ; t\right)\right]\right\},
\end{aligned}
$$

where $\sum^{*}$ is the sum over sets $J_{1}, \ldots, J_{j}$ such that at least two of them have non-void intersection and

$$
\begin{gathered}
\gamma\left(z_{r}, h_{r}, J_{r} ; t\right)=\chi\left(A_{r}\right) \xi_{r}\left(\omega, z_{r}\right) \\
A_{r}=\left\{\omega: \operatorname{cl}(r)=J_{r}, n\left(\bar{J}_{r}=h_{r}\right)\right\} \\
\xi_{r}\left(\omega, z_{r}\right)=\int \mathrm{d} Z_{J_{r}} \mu_{0}^{h_{r}+1}\left(T_{\omega}^{-t} Z_{\bar{J}_{r}}\right) .
\end{gathered}
$$

We start by a bound on $\mathscr{T}_{1}^{j}$. It is:

Now we have, using (5.13):

$$
\begin{aligned}
\left\|\mathscr{T}_{1}^{j}\right\|_{L_{1}} & \leq \sum_{h_{1}, \ldots, h_{j}} \sum_{\substack{J_{1}, \ldots, J_{j} \\
\left|J_{i}\right|=h_{i}}}^{*} \prod_{r=1}^{j} \mathbf{E}\left[\chi\left(A_{r}\right)\right] \\
& \leq \sum_{h_{1}, \ldots, h_{j}} \sum_{r, s} \sum_{\substack{J_{1}, \ldots, J_{j} \\
\text { J } J_{i} \mid=h_{i} \\
J_{r} \cap J_{s}=\phi}} \prod_{r=1}^{j} \mathbf{E}\left[\chi\left(A_{r}\right)\right] .
\end{aligned}
$$

$$
\begin{aligned}
& \sum_{h_{r}, h_{s}} \sum_{\substack{J_{r}, J_{s} \\
\left|J_{i}\right|=h_{i}, i=r, s \\
J_{r} \cap J_{s} \neq \phi}} \mathbf{E}\left[\chi\left(A_{r}\right)\right] \mathbf{E}\left[\chi\left(A_{s}\right)\right] \\
& \leq \sum_{h_{r}, h_{s}} \sum_{\substack{J_{r} \\
\left|J_{r}\right|=h_{r}}} \sum_{i \in J_{r}} \sum_{\substack{J_{s}: i \in J_{s} \\
\left|J_{s}\right|=h_{s}}} \mathbf{E}\left[\chi\left(A_{r}\right)\right] \mathbf{E}\left[\chi\left(A_{s}\right)\right] \\
& \leq \sum_{h_{r}, h_{s}}\left(\begin{array}{l}
N \\
h_{r}
\end{array}\right)\left(\begin{array}{c}
N \\
h_{s}-1
\end{array}\right) h_{r} h_{r} ! h_{s} ! c^{h_{r}+h_{s}} \varepsilon^{h_{r}+h_{s}} \\
& \leq \frac{1}{N} \sum_{h_{r}, h_{s}} h_{s} h_{s} \frac{(N !)^{2}}{\left(N-h_{r}\right) !\left(N-h_{s}+1\right) !} \frac{1}{N^{h_{r}}} \frac{1}{N^{h_{s}-1}}(c \lambda)^{h_{r}+h_{s}} \\
& \leq \frac{1}{N}\left(\sum_{h} h(c \lambda)^{h}\right)^{2} \leq \frac{c^{2}}{N} \text {. }
\end{aligned}
$$


Hence, by (5.33) and again (5.13):

$$
\left\|\mathscr{T}^{j}\right\|_{L_{1}} \leq \frac{c^{2}}{N} j^{2}\left(\sum_{h_{r}} \sum_{\substack{I_{r} \\\left|I_{r}\right|=h_{r}}} \mathbf{E}\left[\chi\left(A_{r}\right)\right]\right)^{j-2} \leq \frac{c^{j}}{N}
$$

Let us pass to $\mathscr{T}_{2}^{j}$. To simplify the algebra we consider for the moment $j=2$. We set, for $I, J \subset I_{N}$,

$$
\Omega(I, J)=\{\omega(i, k): i \in I, k \in J\} .
$$

First of all we notice that the function $\xi_{r}$ in (5.32) depends on $\omega$ through its restriction on $\Omega\left(\bar{J}_{r}, \bar{J}_{r}\right)$ and we will denote such restriction still by $\xi_{r}$. Now

$$
\begin{aligned}
\mathbf{E}\left[\gamma\left(z_{r}, h_{r}, J_{r} ; t\right)\right]= & \sum_{\omega_{r} \in \Omega\left(\bar{J}_{r}, \bar{J}_{r}\right)} p\left(\omega_{r}\right) \xi_{r}\left(\omega_{r}, z_{r}\right) \chi\left(\bar{A}_{r}\right) \\
& \times \sum_{\eta_{r} \in \Omega\left(\bar{J}_{r}, \bar{J}_{r}^{c}\right)} p\left(\eta_{r}\right) \chi\left(\eta_{r}=0\right)
\end{aligned}
$$

for $r=1,2$, where

$$
\bar{A}_{r}=\left\{\omega_{r} \in \Omega\left(\bar{J}_{r}, \bar{J}_{r}\right): \operatorname{cl}(r)=J_{r}, n\left(\bar{J}_{r}\right)=h_{r}\right\} .
$$

The sum over $\eta_{r}$ in (5.37) can be computed, giving $(1-\varepsilon)^{h_{r}\left(N-h_{r}\right)}$. On the other side

$$
\begin{aligned}
\mathbf{E}\left[\gamma\left(z_{1}, h_{1}, J_{1} ; t\right) \gamma\left(z_{2}, h_{2}, J_{2} ; t\right)\right] & p \sum_{\omega_{1} \in \Omega\left(\bar{J}_{1}, \bar{J}_{1}\right)} p\left(\omega_{1}\right) \xi_{1}\left(\omega_{1}\right) \chi\left(\bar{A}_{1}\right) \sum_{\omega_{2} \in \Omega\left(\bar{J}_{2}, \bar{J}_{2}\right)} p\left(\omega_{2}\right) \xi_{2}\left(\omega_{2}\right) \chi\left(\bar{A}_{2}\right) \\
& \times \sum_{\eta \in \Omega\left(\bar{J}_{1} \cup \bar{J}_{2},\left(\bar{J}_{1} \cup \bar{J}_{2}\right)^{c}\right)} p(\eta) \chi(\eta=0) \sum_{\gamma \in \Omega\left(\bar{J}_{1} \bar{J}_{2}\right)} p(\gamma) \chi(\gamma=0) .
\end{aligned}
$$

The last two sums in (5.39) give $(1-\varepsilon)^{\left(h_{1}+h_{2}\right)\left(N-h_{1}-h_{2}\right)}(1-\varepsilon)^{h_{1} h_{2}}$ and by (5.38):

$$
\begin{aligned}
& \mathbf{E}\left[\chi\left(A_{1}\right) \xi_{1}\left(\omega, z_{1}\right) \chi\left(A_{2}\right) \xi_{2}\left(\omega, z_{2}\right)\right] \\
& \quad=\mathbf{E}\left[\chi\left(A_{1}\right) \xi_{1}\left(\omega, z_{1}\right)\right] \mathbf{E}\left[\chi\left(A_{2}\right) \xi_{2}\left(\omega, z_{2}\right)\right](1-\varepsilon)^{-h_{1} h_{2}} .
\end{aligned}
$$

Hence, inserting (5.40) in (5.29) we obtain

$$
\left\|\mathscr{T}_{2}^{2}\right\|_{L_{1}} \leq \sum_{h_{1}, h_{2}} \sum_{J_{1}, J_{2}} \prod_{r=1}^{2} \mathbf{E}\left[\chi\left(A_{r}\right)\right]\left[(1-\varepsilon)^{-h_{1} h_{2}}-1\right]
$$

Noticing that

$$
\begin{aligned}
(1-\varepsilon)^{-h_{1} h_{2}}-1 & \leq \frac{\varepsilon h_{1} h_{2}}{(1-\varepsilon)^{h_{1} h_{2}+1}} \leq 2 \varepsilon h_{1} h_{2}\left(1-\frac{\lambda}{N}\right)^{-N \frac{h_{1} h_{2}}{N}} \\
& \leq \frac{2 \lambda h_{1} h_{2}}{N} e^{\lambda h_{1}}
\end{aligned}
$$

proceeding as usual we obtain, by (5.41), (5.42) and (5.13):

$$
\left\|\mathscr{T}_{2}^{2}\right\|_{L_{1}} \leq \frac{1}{N}\left(\sum_{h \geq 0}(c \lambda)^{h}\right)^{2} \leq \frac{c}{N}
$$


For the term $\mathscr{T}_{2}^{3}$ we proceed analogously and prove that

$$
\mathbf{E}\left[\prod_{r=1}^{j} \chi\left(A_{r}\right) \xi_{r}\right]-\prod_{r=1}^{j} \mathbf{E}\left[\chi\left(A_{r}\right) \xi_{r}\right]=\prod_{r=1}^{j} \mathbf{E}\left[\chi\left(A_{r}\right) \xi_{r}\right]\left[(1-\varepsilon)^{-\prod_{r<s} h_{r} h_{s}}-1\right] .
$$

Following (5.42):

$$
(1-\varepsilon)^{-\prod_{r<s} h_{r} h_{s}}-1 \leq \frac{2 \lambda h^{2}}{N} e^{\lambda h}, \quad h=\sum_{r=1}^{J} h_{r},
$$

we can conclude that

$$
\left\|\mathscr{T}_{2}^{\jmath}\right\|_{L_{1}} \leq \frac{c^{\jmath}}{N}
$$

To achieve the proof of the proposition we have to show that (4.5) holds. Indeed, proceeding as in the proof of estimate (5.26), by the use of Proposition 3.1 we can conclude that

$$
\int_{0}^{\infty} \mathrm{d} t \int \mathscr{T}_{r}^{2}\left(x, v_{1}, x, v_{2} ; t\right) \mathrm{d} x \leq \frac{c}{N}, \quad r=1,2 .
$$

\section{From Small to Large $\lambda$}

In this section we remove the assumption on the smallness of $\lambda$ we used so far. In doing this we pay a price: the quality of the convergence we are able to prove is weaker. This is, probably, a technical limitation only.

The idea underlying this extension is the following. Look at the particle system at time $t+\Delta t$, with $\Delta t$ sufficiently small, and at a small interval $\Lambda$ on the line. Due to the boundedness of the velocities, the particles inside $\Lambda$ are influenced only by the particles in an interval, say $\Lambda_{0}$, a little bit larger than $\Lambda$, if $\Delta t$ is small. Suppose that at the time $t$ we have convergence to the solution of the Boltzmann equation. This means that, with large probability, the number of particles in $\Lambda_{0}$, say $N\left(\Lambda_{0}\right)$, is of the order $\alpha N$, where $\alpha=\sum_{v} \int_{\Lambda_{0}} \mathrm{~d} x f(x, v ; t)$. Since we have $L_{\infty}$ bounds for $f, \alpha$ can be made arbitrarily small, provided that $\Lambda$ and $\Delta t$ are sufficiently small. They will be fixed in such a way that $\alpha \lambda<\lambda_{0}$, where $\lambda_{0}$ is the value under which the cluster expansion is convergent and $\lambda=\varepsilon N$ (in general larger than $\lambda_{0}$ ). On the other hand the particle system at time $t+\Delta t$ restricted in $\Lambda$ is the same as if the particles outside $\Lambda_{0}$ were absent at time $t$, so that we are in a situation for which the number of particles is $\alpha N$ and consequently the effective $\lambda$ is $\alpha N \varepsilon=\alpha \lambda<\lambda_{0}$.

Therefore, using the previous analysis, we expect convergence at time $t+\Delta t$, if we look at the region $\Lambda$. Then it is not difficult to show that the local convergence in each region $\Lambda$ of small size implies the convergence in any region, so that all the argument can be iterated to reach an arbitrary time, since $\Delta t$ is a priori fixed.

To make rigorous the above argument we need a local characterization of a physical state of the system (and we shall do it later) and the lemma below:

Lemma 6.1. Let $\left\{\mu^{N}\right\}_{N>1}$ be a sequence of densities of symmetric probability measures on $(\mathbf{R} \times V)^{N}$. Denote by $f_{j}^{N}$ their marginals. Then the following two statements are equivalent: 
i) For all $j$ and all $\varphi$ bounded, $\varphi: \mathbf{R} \times V \rightarrow \mathbf{R}$,

$$
\int f_{\jmath}^{N}\left(Z_{\jmath}\right) \prod_{i=1}^{\jmath} \varphi\left(Z_{\imath}\right) \mathrm{d} Z_{\jmath} \underset{N \rightarrow \infty}{\longrightarrow}\langle f, \varphi\rangle^{j}
$$

with $f \in L_{1}(\mathbf{R} \times V)$.

ii)

$$
\lim _{N \rightarrow \infty} \mathbf{E}_{N}\left(\left\langle\nu^{N}, \varphi\right\rangle^{j}\right)=\langle f, \varphi\rangle^{\jmath}
$$

Here $\langle\cdot\rangle$ denotes the scalar product, $\left\langle\nu^{N}, \varphi\right\rangle=\frac{1}{N} \sum_{i=1}^{N} \varphi\left(z_{i}\right)$, and $\mathbf{E}_{N}$ is the expectation with respect to $\mu^{N}$.

Proof. By definition, for $N>j$ :

$$
\begin{aligned}
\mathbf{E}_{N}\left(\left\langle\nu^{N}, \varphi\right\rangle^{j}\right)= & \int \mathrm{d} \mu^{N} \frac{1}{N^{\jmath}} \sum_{i_{1}=1}^{N} \ldots \sum_{i_{j}=1}^{N} \varphi\left(z_{i_{1}}\right) \ldots \varphi\left(z_{i_{j}}\right) \\
= & \frac{N(N-1) \ldots(N-j+1)}{N^{j}} \\
& \times \int \mathrm{d} z_{j} f_{j}^{N}\left(z_{j}\right) \prod_{i=1}^{j} \varphi\left(z_{i}\right)+O\left(\frac{1}{N}\right) .
\end{aligned}
$$

Indeed the first term in the right-hand side fof (6.1) arises from the contribution in the sums in which all the $i_{k}$, s are different and the second one from the contribution in which at least two indices coincide.

We now establish a proposition which, combined with the results of the previous section, allows us to prove the convergence for any $\lambda$.

Consider now our particle system distributed, at time zero, according to a sequence of symmetric densities $\left\{\mu^{N}\right\}_{N \geq 1}$ such that, for all bounded continuous $\varphi$ of compact support and for all $j$ :

$$
\mathbf{E}_{N}\left(\left\langle\nu^{N}, \varphi\right\rangle^{j}\right) \underset{N \rightarrow \infty}{\longrightarrow}\langle f, \varphi\rangle^{j}
$$

with $\|f\|_{L_{\infty}} \leq M$.

Proposition 6.2. Assume hypothesis (6.2) and consider an interval $\Lambda$ with $|\Lambda|=$ length of $\Lambda=\delta, \delta=\frac{\lambda_{0}}{100 \lambda M}$ (here $\lambda_{0}$ is a value for which the cluster expansion is converging and $\lambda=\varepsilon N)$. Define

$$
\Lambda_{0}=\{x \pm 2 T \mid x \in \Lambda\}, \quad T=\delta .
$$

Then, for all $\eta>0$ sufficiently small, $Z_{\jmath} \in(\Lambda \times V)^{\jmath}, t \leq T$ the following hold:

$$
\Omega_{j}^{N}\left(Z_{j} t\right)+l_{\jmath}^{N,-}\left(Z_{j}, t\right) \leq f_{j}^{N}\left(Z_{j}, t\right) \leq \Omega_{j}^{N}\left(Z_{j}, t\right)+l_{j}^{N,+}\left(Z_{j}, t\right),
$$

where $\Omega_{j}^{N}$ is a positive function such that:

$$
\int_{(\Lambda \times V)^{j}} \Omega_{\jmath}^{N}\left(Z_{\jmath}, t\right) \varphi_{\jmath}\left(Z_{\jmath}\right) \mathrm{d} Z \underset{N \rightarrow \infty}{\longrightarrow} 0
$$


for all bounded continuous $\varphi_{j}:(\Lambda \times V)^{j} \rightarrow \mathbf{R}$ and

$$
l_{\jmath}^{N, \pm}\left(Z_{j}, t\right)=\int \mathrm{d} Z_{[(\alpha \pm \eta) N]-\jmath} \mathbf{E}\left(\gamma^{[(\alpha \pm \eta) N]}\left(T_{\omega}^{-t} Z_{[(\alpha \pm \eta) N]}\right)\right)
$$

being $[a]$ the integer part of $a$ and

$$
\gamma^{k}\left(Z_{k}\right)=\chi\left(Z_{k} \in\left(\Lambda_{0} \times V\right)^{k}\right) \prod_{i=1}^{k} f\left(z_{i}\right) \cdot \alpha^{-k}
$$

with

$$
\alpha=\int_{\Lambda_{0} \times V} \mathrm{~d} z f(z)
$$

Before giving the proof of Proposition 6.2 we discuss its meaning and consequences.

From Proposition 6.2 we see that the $j$-particle distributions $f_{\jmath}^{N}(\cdot, t)$, for $t$ sufficiently small, are controlled, in the region $(\Lambda \times V)^{j}$, by the $j$-particle distributions $l_{j}^{N, \pm}$ of a particle system whose initial distribution is $\gamma^{N}$, i.e. a product measure supported in $\Lambda_{0}$, associated to a system of $H=[(\alpha \eta) N]$ particles.

Notice that

$$
\alpha N \varepsilon \leq \lambda 4 M(\delta+4 \delta)<\lambda_{0}
$$

so that, for a sufficiently small $\eta$, (for instance $\eta<\lambda_{0} / 2 \lambda$ ) the cluster expansion, associated to the initial measure $\gamma^{H}$, is convergent. By Theorem 4.1:

$$
\lim _{N \rightarrow \infty} l_{j}^{N, \pm}=\left(h^{ \pm}\right)^{\otimes \jmath}
$$

in $L_{1}(\mathbf{R} \times V) .\left(h^{ \pm}\right)$is the solution of the Boltzmann equation (2.14) with initial datum $h_{0}=h^{ \pm}(t=0)=\chi_{\Lambda_{0}}(x) f(x, v) \alpha^{-1}$ and $\lambda$ replaced by $\lambda(\alpha \pm \eta)$. Since $\eta$ is arbitrary, we proved:

$$
\lim _{N \rightarrow \infty} \int_{(\Lambda \times V)^{j}} \mathrm{~d} z_{j} f_{j}^{N}\left(z_{j}, t\right) \varphi_{j}\left(z_{\jmath}\right)=\int_{(\Lambda \times V)^{j}} \mathrm{~d} z_{j} h^{\otimes j}\left(z_{\jmath}, t\right) \varphi_{j}\left(z_{\jmath}\right)
$$

for all continuous bounded $\varphi_{j}:(\Lambda \times V)^{j} \rightarrow \mathbf{R}$, where $h(t)$ solves Eq. (2.14) with $\lambda$ replaced by $\alpha \lambda$ and initial datum $h_{0}$ (indeed, thanks to the $L_{\infty}$ bounds, it is not difficult to show that $h(t)$ is $L_{1}$-continuous with respect to $\lambda$ ). Finally by a simple scaling argument and by virtue of the boundedness of the velocities we conclude that $h(x, v, t)=f(x, v, t)$ for $x \in \Lambda$ and $t \leq T$. Therefore we proved the local convergence:

$$
\lim _{N \rightarrow \infty} \int_{(\Lambda \times \Lambda)^{\jmath}} \mathrm{d} z_{j} f_{j}^{N}\left(Z_{j}, t\right) \varphi_{j}\left(Z_{j}\right)=\int_{(\Lambda \times V)^{\jmath}} \mathrm{d} Z_{j} f^{\otimes \mathcal{J}}\left(z_{j}, t\right) \varphi_{j}\left(Z_{j}\right)
$$

for all intervals $\Lambda$ such that $|\Lambda|<\delta$ and all $\varphi_{j}:(\Lambda \times V)^{j} \rightarrow \mathbf{R}$ continuous and bounded.

We now show how convergence (6.2), assumed at time zero, also holds at time $T$. Indeed take $\varphi: \mathbf{R} \times V \rightarrow \mathbf{R}$, continuous bounded, supported in $\Lambda$ with $|\Lambda|<2 \delta$. Let 
$\Lambda_{i},\left|\Lambda_{i}\right|<\delta, i=1,2$ be two disjoint intervals such that $\Lambda_{1} \cup \Lambda_{2}=\Lambda$, and $\varphi_{i}$ the restriction of $\varphi$ to $\Lambda_{i}$. Then:

$$
\begin{aligned}
& \left|\mathbf{E}_{N}\left(\left\langle\nu^{N}, \varphi\right\rangle^{j}-\langle f, \varphi\rangle^{j}\right)\right| \\
& =\left|\sum_{k=0}^{j}\left(\begin{array}{l}
j \\
k
\end{array}\right) \mathbf{E}_{N}\left(\left\langle\nu^{N}, \varphi_{1}\right\rangle^{k}\left\langle\nu^{N}, \varphi_{2}\right\rangle^{\jmath-k}-\left\langle f, \varphi_{1}\right\rangle^{k}\left\langle f, \varphi_{2}\right\rangle^{\jmath-k}\right)^{j}\right| \\
& \leq \leq \sum_{k=0}^{j}\left(\begin{array}{l}
j \\
k
\end{array}\right)\left\{\left|\mathbf{E}_{N}\left(\left\langle\nu^{N}, \varphi_{1}\right\rangle^{k}\left(\left\langle\nu^{N}, \varphi_{2}\right\rangle^{j-k}-\left\langle f, \varphi_{2}\right\rangle^{j-k}\right)\right)\right|\right. \\
& \left.\quad+\left\langle f, \varphi_{2}\right\rangle^{j-k}\left|\mathbf{E}_{N}\left(\left\langle\nu^{N}, \varphi_{1}\right\rangle^{k}-\left\langle f, \varphi_{1}\right\rangle^{k}\right)\right|\right\} .
\end{aligned}
$$

By Lemma 6.1 and (6.8), the $2^{\text {nd }}$ term in the curl bracket vanishes as $N \rightarrow \infty$. The first term is bounded by:

$$
\begin{aligned}
& \mathbf{E}_{N}\left(\left\langle\nu^{N}, \varphi_{1}\right\rangle^{2 k}\right)^{1 / 2}\left(\mathbf{E}_{N}\left(\left\langle\nu^{N}, \varphi_{2}\right\rangle^{2(j-k)}\right)+\left\langle f, \varphi_{2}\right\rangle^{2(\jmath-k)}\right. \\
& \quad-2\left\langle f, \varphi_{2}\right\rangle^{j-k} \mathbf{E}_{N}\left(\left\langle\nu^{N}, \varphi_{2}\right\rangle^{j-k}\right)^{1 / 2}
\end{aligned}
$$

Also (6.10) vanishes as $N \rightarrow \infty$. In fact, for all $j, E_{N}\left(\left\langle\nu^{N}, \varphi_{2}\right\rangle^{j}\right) \rightarrow\left\langle f, \varphi_{2}\right\rangle^{\jmath}$, again by Lemma 6.1 and (6.8), so that (6.2) holds at time $T$, because, using the above argument, we can deal with any bounded continuous function $\varphi$ of compact support. Finally, since the time $T$ is a priori fixed, we can iterate the argument to prove:

Theorem 6.3. Suppose $f_{0} \in L_{\infty}$ be an initial datum for the Boltzmann equation (2.14) and let $f=f(x, v, t)$ be the unique solution satisfying

$$
\sup _{0 \leq t \leq t^{*}} \sup _{x, v} f(x, v, t) \leq M
$$

for an arbitrary (but fixed) $t^{*}>0$, with $M$ a suitable positive constant depending on $f_{0}$ and $t^{*}$. Let $\mu_{0}^{N}=f_{0}^{\otimes N}$ be the initial condition for the particle system and $\lambda=\varepsilon N$ arbitrary. Then:

$$
\lim _{N \rightarrow \infty}\left\langle f_{j}^{N}(t), \varphi_{j}\right\rangle=\left\langle f^{\otimes \jmath}(t), \varphi_{j}\right\rangle
$$

for all $j$ and all continuous $\varphi_{j}:(\mathbf{R} \times V)^{j} \rightarrow \mathbf{R}$ of compact support.

Remark 1 . The $L_{1}$ convergence of the previous section has been replaced by the weak convergence (6.12). Actually (6.12) has been proved for factorizing test functions $\varphi_{\jmath}$, but it can be recovered by a standard approximation argument.

Remark 2. In the above theorem we used the existence and uniqueness of the solution $f \in L_{\infty}\left(\left[0, t^{*}\right] ; L_{\infty}(\mathbf{R} \times V)\right)$ just to control the number of particles (uniformly in time up to an arbitrary fixed time) in an interval of a given size. This can also be done differently by means of the $H$-theorem. Indeed it is easy to show that the Entropy per particle

$$
H^{N}(t)=\frac{1}{N} \int \mathrm{d} Z_{N} \mu^{N}(t) \log \mu^{N}(t)
$$

is a decreasing function of time. By the subaddivitity of the entropy:

$$
\begin{aligned}
\int f_{1}^{N}(z, t) \log f_{1}^{N}(z, t) \mathrm{d} z & \leq H^{N}(t) \leq H^{N}(0) \\
& =\int f_{0} \log f_{0} \mathrm{~d} z \leq C<+\infty
\end{aligned}
$$


From the above inequality we obtain

$$
\int_{\Lambda} f_{1}^{N}(z, t) \rightarrow 0 \quad \text { as } \quad|\Lambda| \rightarrow 0
$$

uniformly in time.

This observation, combined with the remark in Sect. 4 allows us to avoid to assume the existence of the limit solution, but just to prove it, together with the validity proof.

Remark 3. It is not difficult, at this point, to deal with a particle system in a bounded box with suitable (for instance periodic) boundary conditions. We do not give here the details to avoid an even longer and heavy exposition.

Before proving Proposition 6.2 we need some preliminary definitions.

Let $\Lambda_{0} \subset \mathbf{R}^{1}$ be a bounded interval and $\mu^{N}$ be a distribution density of a $N$-particle system.

Denote (for $\left.Z_{n} \in\left(\Lambda_{0} \in V\right)^{n}\right)$ by:

$$
\mu_{\Lambda_{0}}^{N, n}\left(Z_{n}\right)=\left(\begin{array}{c}
N \\
n
\end{array}\right) \int_{\left(\Lambda_{0} \times V\right)^{N-n}} \mathrm{~d} Z_{N-n} \mu^{N}\left(Z_{N}\right),
$$

the probability density of finding $n$ particles in $\Lambda_{0}$ in the configuration $Z_{n}$ and by

$$
p_{\Lambda_{0}}^{N}(n)=\int_{\left(\Lambda_{0} \times V\right)^{n}} \mathrm{~d} Z_{n} \mu_{\Lambda_{0}}^{N, n}\left(Z_{n}\right)
$$

the probability of finding $n$ particles in $\Lambda_{0}$.

Finally we set

$$
\mu_{\Lambda_{0}}^{N}\left(Z_{n} \mid n\right) \mu_{\Lambda_{0}}^{N, n}\left(Z_{n}\right) \frac{1}{p_{\Lambda_{0}}^{N}(n)}
$$

which is the conditional probability (of having $n$ particles in $\Lambda_{0}$ ) density and

$$
\Phi_{\Lambda, 0}^{N, k}\left(Z_{k} \mid n\right)=\int_{\left(\Lambda_{0} \times V\right)^{n-k}} \mathrm{~d} Z_{n-k} \mu_{\Lambda_{0}}^{N}\left(Z_{n} \mid n\right)
$$

its marginals.

Proof of Proposition 6.2. Fix $\varphi_{j}:(\Lambda \times V)^{j} \rightarrow \mathbf{R}^{+}$continuous and bounded and $t \geq T$. Then

$$
\left\langle\varphi_{j}, f_{j}^{N}(t)\right\rangle \equiv \int \varphi_{\jmath}\left(Z_{j}\right) f_{j}^{N}\left(Z_{j}, t\right) \mathrm{d} Z_{\jmath}=\int \mathrm{d} Z_{N} \mu^{N}\left(Z_{N}\right) \mathbf{E}\left(\varphi\left(T_{\omega}^{t} Z_{N}\right)_{\jmath}\right)
$$

(where $\left(Z_{N}\right)_{j}$ denotes the first $j$ particles of $Z_{N}$ and $\mu^{N}$ is the initial sequence for which (6.2) holds)

$$
=\sum_{k \geq j} p_{\Lambda_{0}}^{N}(k) \int_{\left(\Lambda_{0}\right)^{k}} \mathrm{~d} Z_{k} \mu_{\Lambda_{0}}^{N}\left(Z_{k} \mid k\right) \Psi\left(Z_{k}\right),
$$

where we set

$$
\Psi\left(Z_{k}\right)=\mathbf{E}\left(\varphi\left(T_{\omega}^{t} Z_{k}\right)_{\jmath}\right)
$$


since the first $j$ particles of $T_{\omega}^{t} Z_{N}$ depend on the particles of $Z_{N}$ which are in $\Lambda_{0}$ only.

We now estimate the probability of having a number of particles in $\Lambda_{0}$ (such a random variable is denoted by $N\left(\Lambda_{0}\right)$ ) much different than $\alpha N$. We have, by Tchebichev inequality:

$$
\begin{aligned}
\sum_{|k-\alpha N| \geq \beta N} p_{\Lambda_{0}}^{N}(k) & =\operatorname{Prob}\left(\left|N\left(\Lambda_{0}\right)-\alpha N\right| \geq \beta N\right) \\
& \leq\left(\frac{1}{\beta N}\right)^{2} \mathbf{E}_{N}\left(\left|N\left(\Lambda_{0}\right)-\alpha N\right|^{2}\right) \\
& =(\beta N)^{-2}\left\{\mathbf{E}\left(N\left(\Lambda_{0}\right)^{2}\right)+\alpha^{2} N^{2}-2 \alpha N \mathbf{E}_{N}\left(N\left(\Lambda_{0}\right)\right)\right\}
\end{aligned}
$$

Here $\mathbf{E}_{N}$ denotes the expectation with respect to $\mu_{N}$. By (6.2) $\frac{\mathbf{E}_{N}\left(N\left(\Lambda_{0}\right)^{2}\right)}{N^{2}} \rightarrow \alpha^{2}$, $\frac{\mathbf{E}_{N}\left(N\left(\Lambda_{0}\right)\right)}{N} \rightarrow \alpha$ as $N \rightarrow \infty$, so that the right-hand side of $(6.21)$ is bounded by $\frac{\xi_{1}(N)}{\beta^{2}}$, where $\xi_{1}(N) \rightarrow 0$ as $N \rightarrow \infty$. In conclusion, denoting from now on by $\xi_{i}(N)$, $i=1,2, \ldots$ vanishing sequences in the limit $N \rightarrow \infty$,

$$
\left\langle\varphi_{\jmath}, f_{\jmath}^{N}(t)\right\rangle=\xi_{2}(N)+\sum_{|k-\alpha N|<\eta N} p_{\Lambda_{0}}^{N}(k) \int_{\left(\Lambda_{0}\right)^{k}} \mathrm{~d} Z_{k} \mu_{\Lambda_{0}}^{N}\left(Z_{k} \mid k\right) \Psi\left(Z_{k}\right)
$$

Observe now that for $k \in[(\alpha-\eta) N,(\alpha+\eta) N]$, with a sufficiently small $\eta$, the cluster expansion is convergent, that is:

$$
\begin{aligned}
\left\langle f_{j}^{N}(t), \varphi_{j}\right\rangle= & \xi_{2}(N)+\sum_{k=[(\alpha-\eta) N]}^{[(\alpha+\eta) N]} p_{\Lambda_{0}}^{N}(k) \sum_{h=1}^{k} \sum_{\substack{I \subset I_{k} \backslash I_{j} \\
|I|=h}} \mathbf{E}\left[\chi\left(\operatorname{cl}\left(I_{j}\right)=I\right)\right. \\
& \left.\times \int \mathrm{d} Z_{I \cup I_{\jmath}} \Phi_{\Lambda_{0}}^{N, h+j}\left(Z_{I \cup I_{\jmath}} \mid k\right) \varphi\left(\left(T_{\omega}^{t} Z_{I \cup I_{j}}\right)_{j}\right)\right]
\end{aligned}
$$

from which we argue that

$$
\xi_{4}(N)+S^{-}(N) \leq\left\langle f_{\jmath}^{N}(t), \varphi_{j}\right\rangle \leq \xi_{3}(N)+S^{+}(N),
$$

where

$$
\begin{aligned}
S^{ \pm}(N)= & \sum_{h=1}^{[(\alpha \pm \eta) N]} \sum_{\substack{I \subset I_{[(\alpha \pm \eta) N]} \backslash I_{\jmath} \\
|I|=h}} \mathbf{E}\left(\chi\left(\operatorname{cl}\left(I_{\jmath}\right)=I\right)\right. \\
& \times \int \mathrm{d} Z_{I \cup I_{\jmath}} \sum_{k>h+\imath} p_{\Lambda_{0}}^{N}(k) \Phi^{N, h+j}\left(Z_{I \cup I_{\jmath}} \mid k\right) \phi\left(\left(T_{\omega}^{t} Z_{I \cup I_{j}}\right)_{j}\right)
\end{aligned}
$$


On the other hand, for a positive function $\varphi$ supported in $\Lambda_{0}$ we have:

$$
\begin{aligned}
\mathbf{E}_{N} & \left(\left(\frac{1}{N} \sum_{i=1}^{N} \varphi\left(z_{i}\right)\right)^{\jmath}\right) \\
= & \sum_{k=1}^{N} \int_{\left(\Lambda_{0} \times V\right)^{k}} \frac{1}{N^{j}} \sum_{\imath_{1} \ldots \imath_{\jmath}} \varphi\left(z_{i_{1}}\right) \ldots \varphi\left(z_{i_{j}}\right) \mu_{\Lambda_{0}}^{N, k}\left(Z_{k}\right) \mathrm{d} Z_{k} \\
= & \sum_{k=\jmath}^{N} p_{\Lambda_{0}}^{N}(k) \frac{k(k-1) \ldots(k-j+1)}{N^{j}} \\
& \times \int_{\left(\Lambda_{0} \times V\right)^{\jmath}} \varphi\left(z_{1}\right) \ldots \varphi\left(z_{\jmath}\right) \Phi^{N, j}\left(Z_{j} \mid k\right) \mathrm{d} Z_{j}+O\left(\frac{1}{N}\right) .
\end{aligned}
$$

We know that $p_{\Lambda_{0}}^{N}(k)$ is concentrated around $\alpha N$ as follows by the arguments after (6.21). In conclusion

$$
\begin{aligned}
(\alpha-\beta)^{j} & \sum_{k=\jmath}^{N} p_{\Lambda_{0}}^{N}(k) \int_{\left(\Lambda_{0} \times V\right)^{\jmath}} \varphi\left(z_{1}\right) \ldots \varphi\left(z_{j}\right) \Phi^{N, j}\left(Z_{j} \mid k\right) \mathrm{d} Z_{\jmath}+\xi_{5}(N) \\
\leq & \mathbf{E}_{N}\left(\left(\frac{1}{N} \sum_{i=1}^{N} \varphi\left(z_{\imath}\right)\right)^{j}\right) \\
\leq & (\alpha+\beta)^{j} \sum_{k=j}^{N} p_{\Lambda_{0}}^{N}(k) \int_{\left(\Lambda_{0} \times V\right)^{\jmath}} \varphi\left(z_{1}\right) \ldots \varphi\left(z_{j}\right) \\
& \times \Phi^{N, j}\left(Z_{j} \mid k\right) \mathrm{d} Z_{j}+\xi_{6}(N) .
\end{aligned}
$$

Choosing a suitable sequence $\beta=\beta_{N} \rightarrow 0$ as $N \rightarrow \infty$, making use of Lemma 6.1 and a standard approximation argument we conclude that:

$$
\left\langle f_{j}^{N}, \varphi_{j}\right\rangle=\alpha^{j} \sum_{k=1}^{N} p_{\Lambda_{0}}^{N}(k) \int_{\left(\Lambda_{0} \times V\right)^{j}} \varphi_{j}\left(Z_{j}\right) \Phi^{N, \jmath}\left(Z_{j} \mid k\right) \mathrm{d} Z_{\jmath}+\xi_{7}(N)
$$

for all $\varphi_{j}$ continuous and supported in $\left(\Lambda_{0} \times V\right)^{j}$.

Inserting Eq. (6.28) in (6.25) we obtain:

$$
\begin{aligned}
S^{ \pm}(N)= & \sum_{h=1}^{[(\alpha \pm \eta) N]} \sum_{\substack{I \subset I_{[(\alpha \pm \eta) N] \backslash I_{\jmath}} \\
|I|=h}} \mathbf{E}\left(\chi\left(\mathrm{cl}\left(I_{\jmath}\right)=I\right) \alpha^{-(\jmath+h)}\right. \\
& \times \int \mathrm{d} Z_{I \cup I_{\jmath}} f_{h+j}^{N}\left(Z_{I \cup I_{\jmath}}\right) \varphi\left(\left(T_{\omega}^{t}\left(Z_{I \cup I_{j}}\right)_{j}\right)+\xi_{8}(N) .\right.
\end{aligned}
$$


Finally, by (6.2) and the dominated convergence theorem, which we can apply thanks to the convergence of the cluster expansion, we obtain:

$$
\begin{aligned}
S^{ \pm}(N)= & \sum_{h=1}^{[(\alpha \pm \eta) N]} \sum_{\substack{I \subset I_{[(\alpha \pm \eta) N] \backslash I_{\jmath}} \\
|I|=h}}\left\{\mathbf { E } \left(\chi\left(\operatorname{cl}\left(I_{j}\right)=I\right) \alpha^{-(j+h)}\right.\right. \\
& \left.\times \int \mathrm{d} z_{I \cup I_{\jmath}} \varphi_{j}\left(\left(T_{\omega}^{-t}\left(Z_{I \cup I_{j}}\right)\right)_{j}\right) f^{\otimes(\jmath+h)}\left(Z_{I \cup I_{\jmath}}\right)\right\}+\xi_{9}^{ \pm}(N) \\
= & \left\langle l_{j}^{N, \pm}, \varphi_{\jmath}\right\rangle+\xi_{9}^{ \pm}(N) .
\end{aligned}
$$

This concludes the proof.

Acknowledgements. During the long period of time in which this work was conceived and developed, we had the chance to discuss with many colleagues and friends. We mention extremely useful discussions with R. Esposito, R. Marra, E. Presutti and R. Salvati Manni. Special thanks are due to Leif Arkeryd who contributed to obtain preliminary results and encouraged us in following the strategy discussed in the present paper.

\section{References}

1. Lanford, O.: Time evolution of large classical systems. Lecture Notes in Physics, Vol. 38, Moser, E.J. (ed.), Berlin, Heidelberg, New York: Springer 1975, pp. 1-111

2. Illner, R., Pulvirenti, M.: Global validity of the Boltzmann equation of a two and three-dimensional rare gas in the vacuum: erratum and improved results. Commun. Math. Phys. 121, 143-146 (1989)

3. Di Perna, R., Lions, P.L.: On the Cauchy problem for the Boltzmann equation: global existence and weak stability. Ann. Math. 130, 321-366 (1989)

4. Cercignani, C.: Recent results in kinetic theory of gases. Rendiconti sem. mat. Università di Torino, Proceedings of Nonlinear hyperbolic equation, pp. 47-64

5. Ianiro, N., Lebowitz, J.L.: Stationary nonequilibrium solutions of model Boltzmann equation. Found. Physics. 15, 531 (1985)

6. Caprino, S., De Masi, A., Presutti, E., Pulvirenti, M.: A derivation of the Broadwell equation. Commun. Math. Phys. 135, 443-465 (1991)

Communicated by J.L. Lebowitz 
